\title{
Approximate controllability of impulsive partial neutral stochastic functional integro-differential inclusions with infinite delay
}

Zuomao Yan ${ }^{1,2^{*}}$ and Fangxia $L^{2}$

${ }^{*}$ Correspondence:

yanzuomao@163.com

${ }^{1}$ School of Mathematics and

Statistics, Lanzhou University, Lanzhou, Gansu 730000, P.R. China

${ }^{2}$ Department of Mathematics, Hexi University, Zhangye, Gansu 734000, P.R. China

\section{Springer}

\begin{abstract}
In this paper, the approximate controllability of partial neutral stochastic functional integro-differential inclusions with infinite delay and impulsive effects in Hilbert spaces is considered. By using Hölder's inequality, stochastic analysis and fixed point strategy with the properties of analytic resolvent operator, a new set of sufficient conditions is formulated, which guarantees the approximate controllability of the nonlinear impulsive stochastic system. The results are obtained under the assumption that the associated linear system is approximately controllable. An example is given to illustrate our results.
\end{abstract}

MSC: $34 \mathrm{~A} 37 ; 60 \mathrm{H} 10 ; 34 \mathrm{~K} 50 ; 93 \mathrm{BO}$

Keywords: approximate controllability; impulsive partial neutral stochastic functional integro-differential inclusions; infinite delay; analytic resolvent operator; fixed point

\section{Introduction}

The study of controllability plays an important role in the control theory and engineering [1]. In recent years, various controllability problems for different kinds of dynamical systems have been investigated in many publications; see [2-6] and the references therein. From the mathematical point of view, the problems of exact and approximate controllability are to be distinguished. However, as proved by Triggiani [7], the concept of exact controllability is usually too strong and has limited applicability. In addition, the fixed point techniques are widely used in studying the controllability problems for nonlinear control systems, approximate controllable systems are more prevalent and very often approximate controllability is completely adequate in applications; see $[8,9]$. So, it is important, in fact necessary, to study the weaker concept of controllability, namely approximate controllability for nonlinear systems. The theory of impulsive partial differential equations or inclusions appears as a natural description of several real processes subject to certain perturbations whose duration is negligible in comparison with the duration of the process. It has seen considerable development in the last decade [10]. In the present literature, many papers are concerned with the results on the approximate controllability of solutions to these systems (see [11-14]). Furthermore, besides impulsive effects, stochastic effects like-

(c) 2015 Yan and Lu; licensee Springer. This is an Open Access article distributed under the terms of the Creative Commons Attribution License (http://creativecommons.org/licenses/by/4.0), which permits unrestricted use, distribution, and reproduction in any medium, provided the original work is properly credited. 
wise exist in real systems. Therefore, impulsive stochastic differential equations describing these dynamical systems subject to both impulse and stochastic changes have attracted considerable attention. Particularly, the papers [15-17] studied the existence of mild solutions for some impulsive neutral stochastic functional integro-differential equations with infinite delay in Hilbert spaces.

Recently, much attention has been paid to the approximate controllability for impulsive partial stochastic differential and integrodifferential systems in a Hilbert space. For example, Sakthivel et al. $[18,19]$ established the approximate controllability of nonlinear firstorder and second-order stochastic infinite-dimensional dynamical systems with impulsive effects in a Hilbert space. Subalakshmi and Balachandran [20] discussed the approximate controllability of nonlinear stochastic impulsive integrodifferential systems. Shen and Sun [21] investigated the approximate controllability of stochastic impulsive functional systems with infinite delay. Zang and Li [22] studied the approximate controllability of fractional impulsive neutral stochastic differential equations with nonlocal conditions. As the generalization of classic impulsive differential equations, impulsive stochastic differential inclusions in Hilbert spaces have attracted the researchers' great interest [23, 24]. Among them, Ren et al. [25] obtained the controllability of impulsive neutral stochastic functional differential inclusions with infinite delay in an abstract space by means of the fixed point theorem for discontinuous multivalued operators due to Dhage. In this paper we study the approximate controllability of a class of impulsive partial neutral stochastic functional integro-differential inclusions with infinite delay in Hilbert spaces of the form

$$
\begin{aligned}
& d\left[x(t)-G\left(t, x_{t}\right)\right] \in A\left[x(t)+\int_{0}^{t} h(t-s) x(s) d s\right] d t+B u(t) d t+F\left(t, x_{t}\right) d w(t), \\
& \quad t \in J=[0, b], t \neq t_{k}, k=1, \ldots, m, \\
& x_{0}=\varphi \in \mathcal{B}, \\
& \Delta x\left(t_{k}\right)=I_{k}\left(x_{t_{k}}\right), \quad k=1, \ldots, m,
\end{aligned}
$$

where the state $x(\cdot)$ takes values in a separable real Hilbert space $H$ with the inner product $\langle\cdot, \cdot\rangle_{H}$ and the norm $\|\cdot\|_{H}$, and $A$ is the infinitesimal generator of a compact, analytic resolvent operator $R(t), t>0$ in $H$. The control function $u \in L^{p}(J, U)$, a Hilbert space of admissible control functions, $p \geq 2$ is an integer, and $B$ is a bounded linear operator from a Banach space $U$ to $H$. The time history $x_{t}:(-\infty, 0] \rightarrow H$ given by $x_{t}(\theta)=x(t+\theta)$ belongs to some abstract phase space $\mathcal{B}$ defined axiomatically in Section 2 . Let $K$ be another separable Hilbert space with the inner product $\langle\cdot, \cdot\rangle_{K}$ and the norm $\|\cdot\|_{K}$. Suppose that $\{w(t): t \geq 0\}$ is a given $K$-valued Wiener process with a covariance operator $Q>0$ defined on a complete probability space $(\Omega, \mathcal{F}, P)$ equipped with a normal filtration $\left\{\mathcal{F}_{t}\right\}_{t \geq 0}$, which is generated by the Wiener process $w$. The initial data $\{\varphi(t):-\infty<t \leq 0\}$ is an $\mathcal{F}_{0}$-adapted, $\mathcal{B}$-valued random variable independent of the Wiener process $w$ with finite second moment. $h(t), t \in$ $J$ is a bounded linear operator, and $F, G, I_{k}(k=1, \ldots, m)$ are given functions to be specified later. Moreover, let $0<t_{1}<\cdots<t_{m}<b$ be prefixed points and the symbol $\Delta x\left(t_{k}\right)=x\left(t_{k}^{+}\right)-$ $x\left(t_{k}^{-}\right)$, where $x\left(t_{k}^{-}\right)$and $x\left(t_{k}^{+}\right)$represent the right and left limits of $x(t)$ at $t=t_{k}$, respectively.

The nonlinear integro-differential equation with resolvent operators serves as an abstract formulation of partial integro-differential equations which arises in many physical phenomena $[26,27]$. The resolvent operator is similar to the semigroup operator for 
abstract differential equations in Banach spaces. However, the resolvent operator does not satisfy semigroup properties. To the best of our knowledge, there are no relevant reports on the approximate controllability of impulsive partial stochastic functional integrodifferential inclusions with infinite delay and resolvent operator in the current paper, which is expressed in the form (1.1)-(1.3). Although the papers [13,14] studied the problem of approximate controllability for nonlinear impulsive neutral differential inclusions with nonlocal conditions, besides the fact that $[13,14]$ applies to the approximate controllability of systems with finite delay, the class of deterministic systems is also different from the one studied here. Further, stochastic systems with infinite delay deserve a study because they describe a kind of system present in the real world. Therefore, it is interesting to study the approximate controllability problems for impulsive stochastic inclusions with infinite delays, which are natural generalizations of controllability concepts for impulsive stochastic equations well known in the theory of infinite dimensional control systems. The existence of solutions to systems is a fundamental premise to carry out a study on approximate controllability. The most common and easily verified conditions to guarantee the existence of a solution are the jump operators $I_{k}(k=1, \ldots, m)$ imposing a completely continuous and Lipschitz condition. In this paper, we are only concerned with the case in which $I_{k}$ $(k=1, \ldots, m)$ are continuous, and we study the approximate controllability of nonlinear impulsive stochastic control systems under the assumption that the associated linear system is approximately controllable. The main tools used in this paper are stochastic analysis, analytic resolvent operators, fractional powers of closed operators and the nonlinear alternative of Leray-Schauder type for multivalued maps due to O'Regan combined with approximation techniques. The known results that appeared in $[13,14,21-25]$ are generalized to the impulsive stochastic inclusions settings and the case of infinite delay. Moreover, the results are also new for deterministic systems with impulsive effects.

The rest of this paper is organized as follows. In Section 2, we introduce some notations and necessary preliminaries. Section 3 verifies the existence of solutions for impulsive stochastic control system (1.1)-(1.3). In Section 4, we establish the approximate controllability of impulsive stochastic control system (1.1)-(1.3). In Section 5, an example is given to illustrate our results. Finally, concluding remarks are given in Section 6.

\section{Preliminaries}

Let $H, K$ be two real separable Hilbert spaces, and we denote by $\langle\cdot, \cdot\rangle_{H},\langle\cdot, \cdot\rangle_{K}$ their inner products and by $\|\cdot\|_{H},\|\cdot\|_{K}$ their vector norms, respectively. Let $(\Omega, \mathcal{F}, P)$ be a complete probability space equipped with a normal filtration $\mathcal{F}_{t}, t \in[0, b]$. Let $w$ be a $Q-W i e n e r$ process on $\left(\Omega, \mathcal{F}_{b}, P\right)$ with the covariance operator $Q$ such that $\operatorname{tr} Q<\infty$. We assume that there exists a complete orthonormal system $\left\{e_{n}\right\}_{n=1}^{\infty}$ in $K$, a bounded sequence of nonnegative real numbers $\left\{\lambda_{n}\right\}_{n=1}^{\infty}$ such that $Q e_{n}=\lambda_{n} e_{n}$, and a sequence $\beta_{n}$ of independent Brownian motions such that

$$
\langle w(t), e\rangle=\sum_{n=1}^{\infty} \sqrt{\lambda_{n}}\left\langle e_{n}, e\right\rangle \beta_{n}(t), \quad e \in K, t \in J
$$

and $\mathcal{F}_{t}=\mathcal{F}_{t}^{w}$, where $\mathcal{F}_{t}^{w}$ is the $\sigma$-algebra generated by $\{w(s): 0 \leq s \leq t\}$. Let $L_{2}^{0}=$ $L_{2}\left(Q^{1 / 2} K ; H\right)$ be the space of all Hilbert-Schmidt operators from $Q^{1 / 2} K$ to $H$ with the inner product $\langle\psi, \theta\rangle_{L_{2}^{0}}=\operatorname{Tr}\left(\psi Q \theta^{*}\right)$. Let $L^{p}\left(\mathcal{F}_{b}, H\right)$ be the Banach space of all $\mathcal{F}_{b}$-measurable 
$p$ th power integrable random variables with values in the Hilbert space $H$. Let $C([0, b]$; $\left.L^{p}(\mathcal{F}, H)\right)$ be the Banach space of continuous maps from $[0, b]$ into $L^{p}(\mathcal{F}, H)$ satisfying the condition $\sup _{t \in J} E\|x(t)\|_{H}^{p}<\infty$.

$L(H, K)$ be the space of bounded linear operators mapping $K$ into $H$ equipped with the usual norm $\|\cdot\|_{H}$, and let $L(H)$ denote the Banach space of bounded linear operators from $H$ to $H$. The notation $B_{r}(x, H)$ stands for the closed ball with center at $x$ and radius $r>0$ in $H$.

In this paper, we assume that the phase space $\left(\mathcal{B},\|\cdot\|_{\mathcal{B}}\right)$ is a semi-normed linear space of $\mathcal{F}_{0}$-measurable functions mapping $(-\infty, 0]$ into $H$ and satisfying the following fundamental axioms due to Hale and Kato (see, e.g., [28]).

(A) If $x:(-\infty, \sigma+b] \rightarrow H, b>0$, is such that $\left.x\right|_{[\sigma, \sigma+b]} \in \mathcal{P C}([\sigma, \sigma+b], H)$ and $x_{\sigma} \in \mathcal{B}$, then for every $t \in[\sigma, \sigma+b]$ the following conditions hold:

(i) $x_{t}$ is in $\mathcal{B}$;

(ii) $\|x(t)\|_{H} \leq \tilde{H}\left\|x_{t}\right\|_{\mathcal{B}}$;

(iii) $\left\|x_{t}\right\|_{\mathcal{B}} \leq K(t-\sigma) \sup \left\{\|x(s)\|_{H}: \sigma \leq s \leq t\right\}+M(t-\sigma)\left\|x_{\sigma}\right\|_{\mathcal{B}}$, where $\tilde{H} \geq 0$ is a constant; $K, M:[0, \infty) \rightarrow[1, \infty), K$ is continuous and $M$ is locally bounded, and $\tilde{H}, K, M$ are independent of $x(\cdot)$.

(B) For the function $x(\cdot)$ in (A), the function $t \rightarrow x_{t}$ is continuous from $[\sigma, \sigma+b]$ into $\mathcal{B}$.

(C) The space $\mathcal{B}$ is complete.

Remark 2.1 ([29]) Let $\varphi \in \mathcal{B}$ and $t \leq 0$. The notation $\varphi_{t}$ represents the function defined by $\varphi_{t}=\varphi(t+\theta)$. Consequently, if the function $x(\cdot)$ in axiom (A) is such that $x_{0}=\varphi$, then $x_{t}=\varphi_{t}$. We observe that $\varphi_{t}$ is well defined for $t<0$ since the domain of $\varphi$ is $(-\infty, 0]$.

Remark 2.2 ([29]) In retarded functional differential equations without impulses, the axioms of the abstract phase space $\mathcal{B}$ include the continuity of the function $t \rightarrow x_{t}$. Due to the impulsive effect, this property is not satisfied in impulsive delay systems and, for this reason, has been eliminated in our abstract description of $\mathcal{B}$.

Let $\mathcal{P}(H)$ denote the class of all nonempty subsets of $H$. Let $\mathcal{P}_{b d, c l}(H), \mathcal{P}_{c p, c v}(H)$, $\mathcal{P}_{b d, c l, c v}(H)$ and $\mathcal{P}_{c d}(H)$ denote respectively the families of all nonempty bounded-closed, compact-convex, bounded-closed-convex and compact-acyclic (see [30]) subsets of $H$. For $x \in H$ and $Y, Z \in \mathcal{P}_{b d, c l}(H)$, we define $D(x, Y)=\inf \left\{\|x-y\|_{H}: y \in Y\right\}$ and $\tilde{\rho}(Y, Z)=$ $\sup _{a \in Y} D(a, Z)$, and the Hausdorff metric $H_{d}: \mathcal{P}_{b d, c l}(H) \times \mathcal{P}_{b d, c l}(H) \rightarrow \mathbb{R}^{+}$by $H_{d}(\tilde{A}, \tilde{B})=$ $\max \{\tilde{\rho}(\tilde{A}, \tilde{B}), \tilde{\rho}(\tilde{B}, \tilde{A})\}$.

$G$ is called upper semicontinuous (u.s.c.) on $H$ if, for each $x_{0} \in H$, the set $G\left(x_{0}\right)$ is a nonempty, closed subset of $H$ and if, for each open set $S$ of $H$ containing $G\left(x_{0}\right)$, there exists an open neighborhood $S$ of $x_{0}$ such that $G(S) \subseteq V$.F is said to be completely continuous if $G(V)$ is relatively compact for every bounded subset $V \subseteq H$.

If the multivalued map $G$ is completely continuous with nonempty compact values, then $G$ is u.s.c. if and only if $F$ has a closed graph, i.e., $x_{n} \rightarrow x_{*}, y_{n} \rightarrow y_{*}, y_{n} \in G\left(x_{n}\right)$ imply $y_{*} \in G\left(x_{*}\right)$.

A multivalued map $G: J \rightarrow \mathcal{P}_{b d, c l, c v}(H)$ is said to be measurable if for each $x \in H$, the function $t \mapsto D(x, G(t))$ is a measurable function on $J$. 
Definition 2.1 Let $G: H \rightarrow \mathcal{P}_{b d, c l}(H)$ be a multivalued map. Then $G$ is called a multivalued contraction if there exists a constant $\kappa \in(0,1)$ such that for each $x, y \in H$ we have

$$
H_{d}(G(x)-G(y)) \leq \kappa\|x-y\|_{H} .
$$

The constant $\kappa$ is called a contraction constant of $G$.

A function $x:[\mu, \tau] \rightarrow H$ is said to be a normalized piecewise continuous function on $[\mu, \tau]$ if $x$ is piecewise continuous and left continuous on $(\mu, \tau]$. We denote by $\mathcal{P C}([\mu, \tau], H)$ the space formed by the normalized piecewise continuous, $\mathcal{F}_{t}$-adapted measurable processes from $[\mu, \tau]$ into $H$. In particular, we introduce the space $\mathcal{P C}$ formed by all $\mathcal{F}_{t}$-adapted measurable, $H$-valued stochastic processes $\{x(t): t \in[0, b]\}$ such that $x$ is continuous at $t \neq t_{k}, x\left(t_{k}\right)=x\left(t_{k}^{-}\right)$and $x\left(t_{k}^{+}\right)$exists for $k=1,2, \ldots, m$. In this paper, we always assume that $\mathcal{P C}$ is endowed with the norm $\|x\|_{\mathcal{P C}}=\left(\sup _{0 \leq t \leq b} E\|x(t)\|^{p}\right)^{\frac{1}{p}}$. Then $\left(\mathcal{P C},\|\cdot\|_{\mathcal{P C}}\right)$ is a Banach space.

To simplify the notations, we put $t_{0}=0, t_{m+1}=b$ and for $x \in \mathcal{P C}$, we denote by $\hat{x}_{k} \in$ $C\left(\left[t_{k}, t_{k+1}\right] ; L_{2}(\Omega, H)\right), k=0,1, \ldots, m$, the function given by

$$
\hat{x}_{k}(t):= \begin{cases}x(t) & \text { for } t \in\left(t_{k}, t_{k+1}\right] \\ x\left(t_{k}^{+}\right) & \text {for } t=t_{k} .\end{cases}
$$

Moreover, for $B \subseteq \mathcal{P C}$ we denote by $\hat{B}_{k}, k=0,1, \ldots, m$, the set $\hat{B}_{k}=\left\{\hat{x}_{k}: x \in B\right\}$.

Let $x_{b}\left(x_{0} ; u\right)$ be the state value of system (1.1)-(1.3) at terminal time $b$ corresponding to the control $u$ and the initial value $x_{0}=\varphi(t) \in \mathcal{B}$. Introduce the set

$$
\mathcal{B}\left(b, x_{0}\right)=\left\{x_{b}\left(x_{0} ; u\right)(0): u(\cdot) \in L^{p}(J, U)\right\}
$$

which is called the reachable set of system (1.1)-(1.3) at terminal time $b$, its closure in $H$ is denoted by $\overline{\mathcal{B}\left(b, x_{0}\right)}$.

Now we give the definitions of mild solutions and approximate controllability for system (1.1)-(1.3).

Definition 2.2 An $\mathcal{F}_{t}$-adapted stochastic process $x:(-\infty, b] \rightarrow H$ is called a mild solution of system (1.1)-(1.3) if $x_{0}=\varphi \in \mathcal{B}$ satisfies $x_{0} \in L_{2}^{0}(\Omega, H),\left.x\right|_{J} \in \mathcal{P C}$, for each $t \in J$, the function $A R(t-s) G\left(s, x_{s}\right), s \in[0, t)$ is integrable and $\Delta x\left(t_{k}\right)=I_{k}\left(x_{t_{k}}\right), k=1, \ldots, m$, such that

$$
\begin{aligned}
x(t)= & R(t)[\varphi(0)-G(0, \varphi)]+\int_{0}^{t} A R(t-s) G\left(s, x_{s}\right) d s \\
& +\int_{0}^{t} A R(t-s) \int_{0}^{s} h(s-\tau) G\left(\tau, x_{\tau}\right) d \tau d s \\
& +\int_{0}^{t} R(t-s) B u(s) d s+\int_{0}^{t} R(t-s) f(s) d w(s) \\
& +\sum_{0<t_{k}<t} R\left(t-t_{k}\right) I_{k}\left(x_{t_{k}}\right), \quad t \in J,
\end{aligned}
$$

where $f \in S_{F, x}=\left\{f \in L^{p}(K, H): f(t) \in F\left(t, x_{t}\right)\right.$ a.e. $\left.t \in J\right\}$. 
Definition 2.3 System (1.1)-(1.3) is said to be approximately controllable on the interval $J$ if $\overline{\mathcal{B}\left(b, x_{0}\right)}=H$.

It is convenient at this point to define operators

$$
\begin{aligned}
& \Gamma_{\tau}^{b}=\int_{\tau}^{b} R(b-s) B B^{*} R^{*}(b-s) d s, \quad 0 \leq \tau<b, \\
& \Gamma_{0}^{b}=\int_{0}^{b} R(b-s) B B^{*} R^{*}(b-s) d s, \\
& R\left(a, \Gamma_{0}^{b}\right)=\left(a I+\Gamma_{0}^{b}\right)^{-1} \quad \text { for } a>0,
\end{aligned}
$$

where $B^{*}$ denotes the adjoint of $B$ and $R^{*}(t)$ is the adjoint of $R(t)$. It is straightforward that the operator $\Gamma_{0}^{b}$ is a linear bounded operator.

(S1) $a R\left(a, \Gamma_{\tau}^{b}\right) \rightarrow 0,0 \leq \tau<s \leq b$, as $a \rightarrow 0^{+}$in the strong operator topology.

Consider the following linear stochastic integro-differential system:

$$
\begin{aligned}
& d x(t)=A\left[x(t)+\int_{0}^{t} h(t-s) x(s) d s\right] d t+B u(t) d t, \quad t \in J=[0, b], \\
& x_{0}=\varphi \in \mathcal{B} .
\end{aligned}
$$

From [8] and [9], we have the following lemma.

Lemma 2.1 Assumption(S1) holds if and only if the linear integro-differential control system (2.1)-(2.2) is approximately controllable on $J$.

The proof of Lemma 2.1 can be performed along the direction of the proof of Theorem 2 in [8].

Lemma 2.2 ([9]) For any $\tilde{x}_{b} \in L^{p}\left(\mathcal{F}_{b}, H\right)$, there exists $\tilde{\phi} \in L_{\mathcal{F}}^{p}\left(\Omega ; L^{2}\left(0, b ; L_{2}^{0}\right)\right)$ such that $\tilde{x}_{b}=$ $E \tilde{x}_{b}+\int_{0}^{b} \tilde{\phi}(s) d w(s)$.

Now, for any $a>0$ and $\tilde{x}_{b} \in L^{p}\left(\mathcal{F}_{b}, H\right)$, we define the control function

$$
\begin{aligned}
u_{x}^{a}(t)= & B^{*} R^{*}(b-t)\left(a I+\Gamma_{0}^{b}\right)^{-1}\left[E \tilde{x}_{b}+\int_{0}^{b} \tilde{\phi}(s) d w(s)\right. \\
& \left.-R(b)[\varphi(0)-G(0, \varphi)]-G\left(b, x_{b}\right)\right] \\
& -B^{*} R^{*}(b-t) \int_{0}^{b}\left(a I+\Gamma_{s}^{b}\right)^{-1} A R(b-s) G\left(s, x_{s}\right) d s \\
& -B^{*} R^{*}(b-t) \int_{0}^{b}\left(a I+\Gamma_{s}^{b}\right)^{-1} A R(b-s) \int_{0}^{s} h(s-\tau) G\left(\tau, x_{\tau}\right) d \tau d s \\
& -B^{*} R^{*}(b-t) \int_{0}^{b}\left(a I+\Gamma_{s}^{b}\right)^{-1} R(b-s) f(s) d w(s) \\
& -B^{*} R^{*}(b-t)\left(a I+\Gamma_{s}^{b}\right)^{-1} \sum_{k=1}^{m} R\left(b-t_{k}\right) I_{k}\left(x_{t_{k}}\right),
\end{aligned}
$$

where $f \in S_{F, x}=\left\{f \in L^{p}(K, H): f(t) \in F\left(t, x_{t}\right)\right.$ a.e. $\left.t \in J\right\}$. 
The next result is a consequence of the phase space axioms.

Lemma 2.3 Let $x:(-\infty, b] \rightarrow H$ be an $\mathcal{F}_{t}$-adapted measurable process such that the $\mathcal{F}_{0}$-adapted process $x_{0}=\varphi(t) \in L_{2}^{0}(\Omega, \mathcal{B})$ and $\left.x\right|_{J} \in \mathcal{P C}(J, H)$, then

$$
\left\|x_{s}\right\|_{\mathcal{B}} \leq M_{b} E\|\varphi\|_{\mathcal{B}}+K_{b} \sup _{0 \leq s \leq b} E\|x(s)\|_{H}
$$

where $M_{b}=\sup _{t \in J} M(t)$ and $K_{b}=\sup _{t \in J} K(t)$.

Lemma 2.4 ([26]) A family of bounded linear operators $R(t) \in \mathcal{P}(H)$ is called a resolvent operator for

$$
\frac{d x}{d t}=A\left[x(t)+\int_{0}^{t} h(t-s) x(s) d s\right]
$$

if

(i) $R(0)=I$ the identity operator on $H$;

(ii) for all $x \in H, R(t) x$ is continuous for $t \in[0, b]$;

(iii) $R(t) \in \mathcal{P}(Y), t \in J$, where $Y$ is the Banach space formed from $D(R)$ endowed with the graph norm. For $y \in Y, R(\cdot) y \in C^{1}(J, H) \cap C(J, Y)$ and

$$
\begin{aligned}
\frac{d x}{d t} R(t) y & =A\left[R(t) y+\int_{0}^{t} h(t-s) R(s) y d s\right] \\
& =R(t) A y+\int_{0}^{t} R(t-s) A h(s) y d s, \quad t \in J .
\end{aligned}
$$

Let $0 \in \rho(A)$, then it is possible to define the fractional power $(-A)^{\alpha}$ for $0<\alpha \leq 1$ as a closed linear operator on its domain $D\left((-A)^{\alpha}\right)$ with inverse $(-A)^{-\alpha}$. Furthermore, the subspace $D\left((-A)^{\alpha}\right)$ is dense in $H$ and the expression $\|x\|_{\alpha}=\left\|(-A)^{\alpha} x\right\|_{H}$ for $x \in D\left((-A)^{\alpha}\right)$ defines a norm on $D\left((-A)^{\alpha}\right)$. Hereafter we denote by $H_{\alpha}$ the Banach space $D\left((-A)^{\alpha}\right)$ normed with $\|\cdot\|_{\alpha}$. Then, for each $0<\alpha \leq 1, H_{\alpha}$ is a Banach space. Furthermore, the following properties are well known [27].

Lemma 2.5 The following properties hold:

(i) If $0<\beta<\alpha \leq 1$, then $H_{\alpha} \subset H_{\beta}$ and the embedding is compact whenever the resolvent operator of $A$ is compact.

(ii) For each $0<\alpha \leq 1$, there exists a positive constant $M_{\alpha}$ such that

$$
\left\|(-A)^{\alpha} R(t)\right\|_{H} \leq \frac{M_{\alpha}}{t^{\alpha}}
$$

Lemma 2.6 $A$ set $B \subseteq \mathcal{P C}$ is relatively compact in $\mathcal{P C}$ if and only if the set $\hat{B}_{k}$ is relatively compact in $C\left(\left[t_{k}, t_{k+1}\right] ; L_{2}(\Omega, H)\right)$ for every $k=0,1, \ldots, m$.

Lemma 2.7 ([31]) For any $p \geq 1$ and for an arbitrary $L_{2}^{0}(K, H)$-valued predictable process $\phi(\cdot)$ such that

$$
\sup _{s \in[0, t]} E\left\|\int_{0}^{s} \phi(v) d w(v)\right\|_{H}^{2 p} \leq(p(2 p-1))^{p}\left(\int_{0}^{t}\left(E\|\phi(s)\|_{L_{2}^{0}}^{2 p}\right)^{1 / p} d s\right)^{p}, \quad t \in[0, \infty) .
$$


Lemma 2.8 (Nonlinear alternative of Leray-Schauder type for multivalued maps due to O'Regan [32]) Let $H$ be a Hilbert space with $V$ an open, convex subset of $H$ and $y \in H$. Suppose

(a) $\Phi: \bar{V} \rightarrow \mathcal{P}_{c d}(H)$ has a closed graph, and

(b) $\Phi: \bar{V} \rightarrow \mathcal{P}_{c d}(H)$ is a condensing map with $\Phi(\bar{V})$, a subset of a bounded set in $H$ holds. Then either

(i) $\Phi$ has a fixed point in $\bar{V}$; or

(ii) there exist $y \in \partial V$ and $\lambda \in(0,1)$ with $y \in \lambda \Phi(y)+(1-\lambda)\left\{y_{0}\right\}$.

\section{Existence of solutions for an impulsive stochastic control system}

In this section, we prove the existence of solutions for impulsive stochastic control system (1.1)-(1.3). We make the following hypotheses.

(H1) The analytic resolvent operator $R(t)$ is compact for all $t>0$, and there exist constants $M, M_{1}, \delta$ such that $\|R(t)\|_{L(H)} \leq M e^{-\delta t},\|h(t)\|_{L(H)} \leq M_{1}$ for every $t \geq 0$.

(H2) The multivalued map $F: J \times \mathcal{B} \rightarrow \mathcal{P}_{b d, c l, c v}(L(K, H))$; for each $t \in J$, the function $F(t, \cdot): \mathcal{B} \rightarrow \mathcal{P}_{b d, c l, c v}(L(K, H))$ is u.s.c.; for each $\psi \in \mathcal{B}$, the function $F(\cdot, \psi)$ is measurable and the set

$$
S_{F, \psi}=\left\{f \in L^{p}(J,(L(K, H))): f(t) \in F(t, \psi) \text { for a.e. } t \in J\right\}
$$

is nonempty.

(H3) There exist a continuous function $m_{f}: J \rightarrow[0, \infty)$ and a continuous nondecreasing function $\Theta_{f}:[0, \infty) \rightarrow(0, \infty)$ such that

$$
\|F(t, \psi)\|_{H}^{p}=\sup \left\{E\|f\|_{H}^{p}: f \in F(t, \psi)\right\} \leq m_{f}(t) \Theta_{f}\left(\|\psi\|_{\mathcal{B}}^{p}\right)
$$

a.e. $t \in J$ and each $\psi \in \mathcal{B}$ with

$$
\int_{1}^{\infty} \frac{1}{s+\Theta_{f}(s)} d s=\infty
$$

(H4) There exists a constant $\beta$ with $0<\beta<1$ such that $G: J \times \mathcal{B} \rightarrow H$ is a continuous function, and $(-A)^{\beta} G: J \times \mathcal{B} \rightarrow H$ satisfies the Lipschitz condition, that is, there exists a constant $L_{G}>0$ such that

$$
E\left\|(-A)^{\beta}\left[G\left(t_{1}, \psi_{1}\right)-G\left(t_{2}, \psi_{2}\right)\right]\right\|_{H}^{p} \leq L_{G}\left[\left|t_{1}-t_{2}\right|+\left\|\psi_{1}-\psi_{2}\right\|_{\mathcal{B}}^{p}\right]
$$

for all $t_{i} \in J, \psi_{i} \in \mathcal{B}, i=1,2$, and

$$
E\left\|(-A)^{\beta} G(t, \psi)\right\|_{H}^{p} \leq L_{G}\left(\|\psi\|_{\mathcal{B}}^{p}+1\right), \quad t \in J, \psi \in \mathcal{B} .
$$

(H5) The functions $I_{k}: \mathcal{B} \rightarrow H$ are continuous and there exist constants $c_{k}$ such that

$$
\limsup _{\|\psi\|_{\mathcal{B}}^{p} \rightarrow \infty} \frac{E\left\|I_{k}(\psi)\right\|_{H}^{p}}{\|\psi\|_{\mathcal{B}}^{p}}=c_{k}
$$

for every $\psi \in \mathcal{B}, k=1, \ldots, m$. 
Lemma 3.1 ([33]) Let J be a compact interval and $H$ be a Hilbert space. Let $F$ be a multivalued map satisfying $(\mathrm{H} 2)$, and let $\tilde{P}$ be a linear continuous operator from $L^{p}(J, H)$ to $C(J, H)$. Then the operator

$$
\tilde{P} \circ S_{F}: C(J, H) \rightarrow \mathcal{P}_{c p, c v}(H), \quad x \rightarrow\left(\tilde{P} \circ S_{F}\right)(x):=\tilde{P}\left(S_{F}, x\right)
$$

is a closed graph in $C(J, H) \times C(J, H)$.

Theorem 3.1 If assumptions (H1)-(H5) are satisfied, then further suppose that for all a $>0$, system (1.1)-(1.3) has at least one mild solution on J, provided that

$$
\begin{aligned}
& 14^{p-1} K_{b}^{p} M^{p}\left[\left(\left\|(-A)^{-\beta}\right\|_{H}^{p}+M_{1-\beta}^{p} \frac{b^{p \beta}}{1-p(1-\beta)}\right.\right. \\
& \left.\left.+M_{1-\beta}^{p} M_{1}^{p} \frac{b^{p(1+\beta)}}{1-p(1-\beta)}\right) L_{G}+m^{p-1} M^{p} \sum_{k=1}^{m} c_{k}\right] N_{*}^{p}<1,
\end{aligned}
$$

where $N_{*}=\max \left\{1, e^{\delta b}\right\}$.

Proof Consider the space $\mathcal{B P C}=\left\{x:(-\infty, b] \rightarrow H ; x_{0}=0,\left.x\right|_{J} \in \mathcal{P C}(J, H)\right\}$ endowed with the uniform convergence topology and define the multivalued map $\Phi: \mathcal{B P C} \rightarrow \mathcal{P}(\mathcal{B P C})$ by $\Phi x$ the set of $\rho \in \mathcal{B P C}$ such that

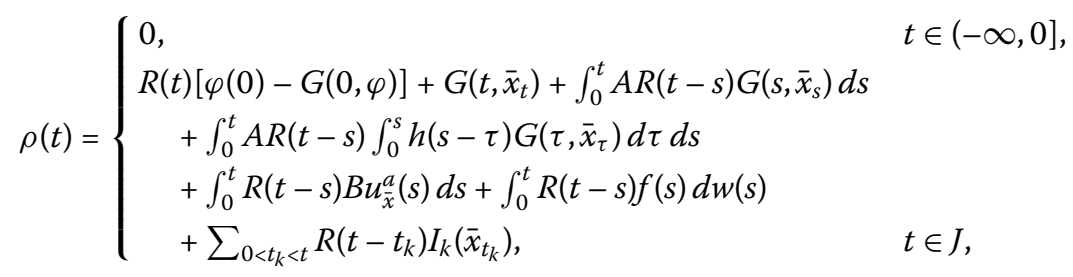

where $f \in S_{F, \bar{x}}=\left\{f \in L^{p}(L(K, H)): f(t) \in F\left(t, \bar{x}_{t}\right)\right.$ a.e. $\left.t \in J\right\}$, and $\bar{x}:(-\infty, 0] \rightarrow H$ is such that $\bar{x}_{0}=\varphi$ and $\bar{x}=x$ on $J$ and

$$
\begin{aligned}
u_{\bar{x}}^{a}(s)= & B^{*} R^{*}(b-s)\left(a I+\Gamma_{0}^{b}\right)^{-1}\left[E \tilde{x}_{b}+\int_{0}^{b} \tilde{\phi}(\eta) d w(\eta)-R(b)[\varphi(0)-G(0, \varphi)]\right. \\
& \left.-G\left(b, x_{b}\right)\right]-B^{*} R^{*}(b-s) \int_{0}^{b}\left(a I+\Gamma_{\eta}^{b}\right)^{-1} A R(b-\eta) G\left(\eta, \bar{x}_{\eta}\right) d \eta \\
& -B^{*} R^{*}(b-s) \int_{0}^{b}\left(a I+\Gamma_{\eta}^{b}\right)^{-1} A R(b-\eta) \int_{0}^{\eta} h(\eta-v) G\left(v, \bar{x}_{v}\right) d v d \eta \\
& -B^{*} R^{*}(b-s) \int_{0}^{b}\left(a I+\Gamma_{\eta}^{b}\right)^{-1} R(b-\eta) f(\eta) d w(\eta) \\
& -B^{*} R^{*}(b-s)\left(a I+\Gamma_{\eta}^{b}\right)^{-1} \sum_{k=1}^{m} R\left(b-t_{k}\right) I_{k}\left(\bar{x}_{t_{k}}\right),
\end{aligned}
$$

where $f \in S_{F, \bar{x}}$. We show that $\Phi$ has a fixed point, which in turn is a mild solution of problem (1.1)-(1.3). 
Let $\bar{\varphi}:(-\infty, 0) \rightarrow H$ be the extension of $(-\infty, 0]$ such that $\bar{\varphi}(\theta)=\varphi(0)=0$ on $J$. Now, let $\left\{\sigma_{n}: n \in \mathbb{N}\right\}$ be a decreasing sequence in $(0, b)$ such that $\lim _{n \rightarrow \infty} \sigma_{n}=0$. To prove the above problem, we consider the following inclusion:

$$
\begin{aligned}
& d\left[x(t)-R\left(\sigma_{n}\right) G\left(t, x_{t}\right)\right] \in A\left[x(t)+\int_{0}^{t} h(t-s) x(s) d s\right] d t+B u(t) d t+F\left(t, x_{t}\right) d w(t), \\
& \quad t \in J=[0, b], t \neq t_{k}, k=1,2, \ldots, m, \\
& x_{0}=\varphi \in \mathcal{B}, \\
& \Delta x\left(t_{k}\right)=R\left(\sigma_{n}\right) I_{k}\left(x_{t_{k}}\right), \quad k=1, \ldots, m,
\end{aligned}
$$

has at least one mild solution $x_{n} \in \mathcal{B P C}$.

For fixed $n \in \mathbb{N}$, set the multivalued map $\Phi_{n}: \mathcal{B P C} \rightarrow \mathcal{P}(\mathcal{B P C})$ defined by $\Phi_{n} x$ the set of $\rho_{n} \in \mathcal{B P C}$ such that

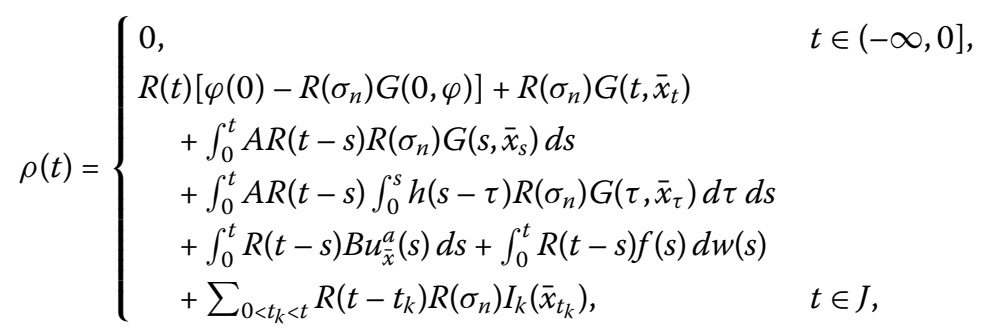

where

$$
\begin{aligned}
u_{\bar{x}}^{a}(s)= & B^{*} R^{*}(b-s)\left(a I+\Gamma_{0}^{b}\right)^{-1}\left[E \tilde{x}_{b}+\int_{0}^{b} \tilde{\phi}(\eta) d w(\eta)-R(b)\left[\varphi(0)-R\left(\sigma_{n}\right) G(0, \varphi)\right]\right. \\
& \left.-R\left(\sigma_{n}\right) G\left(b, x_{b}\right)\right]-B^{*} R^{*}(b-s) \int_{0}^{b}\left(a I+\Gamma_{\eta}^{b}\right)^{-1} A R(b-\eta) R\left(\sigma_{n}\right) G\left(\eta, \bar{x}_{\eta}\right) d \eta \\
& -B^{*} R^{*}(b-s) \int_{0}^{b}\left(a I+\Gamma_{\eta}^{b}\right)^{-1} A R(b-\eta) \int_{0}^{\eta} h(\eta-v) R\left(\sigma_{n}\right) G\left(\nu, \bar{x}_{v}\right) d v d \eta \\
& -B^{*} R^{*}(b-s) \int_{0}^{b}\left(a I+\Gamma_{\eta}^{b}\right)^{-1} R(b-\eta) f(\eta) d w(\eta) \\
& -B^{*} R^{*}(b-s)\left(a I+\Gamma_{\eta}^{b}\right)^{-1} \sum_{k=1}^{m} R\left(b-t_{k}\right) R\left(\sigma_{n}\right) I_{k}\left(\bar{x}_{t_{k}}\right), \quad t \in J
\end{aligned}
$$

where $f \in S_{F, \bar{x}}$. It is easy to see that the fixed point of $\Phi_{n}$ is the mild solution of problem (3.2)-(3.4). We now show that $\Phi_{n}$ satisfies all the conditions of Lemma 2.8. The proof will be given in several steps.

Step 1 . We shall show that there exists an open set $V \subseteq \mathcal{B P C}$ with $x \in \lambda \Phi_{n} x$ for $\lambda \in(0,1)$ and $x \notin \partial V$.

Let $\lambda \in(0,1)$ and let $x \in \lambda \Phi_{n} x$, then there exists $f \in S_{F, \bar{x}}$ such that

$$
\begin{aligned}
x(t)= & \lambda R(t)\left[\varphi(0)-R\left(\sigma_{n}\right) G(0, \varphi)\right]+\lambda R\left(\sigma_{n}\right) G\left(t, \bar{x}_{t}\right) \\
& +\lambda \int_{0}^{t} A R(t-s) R\left(\sigma_{n}\right) G\left(s, \bar{x}_{s}\right) d s
\end{aligned}
$$




$$
\begin{aligned}
& +\lambda \int_{0}^{t} A R(t-s) \int_{0}^{s} h(s-\tau) R\left(\sigma_{n}\right) G\left(\tau, \bar{x}_{\tau}\right) d \tau d s \\
& +\lambda \int_{0}^{t} R(t-s) B\left\{B ^ { * } R ^ { * } ( b - s ) ( a I + \Gamma _ { 0 } ^ { b } ) ^ { - 1 } \left[E \tilde{x}_{b}+\int_{0}^{b} \tilde{\phi}(\eta) d w(\eta)\right.\right. \\
& \left.-R(b)\left[\varphi(0)-R\left(\sigma_{n}\right) G(0, \varphi)\right]-R\left(\sigma_{n}\right) G\left(b, x_{b}\right)\right] \\
& -B^{*} R^{*}(b-s) \int_{0}^{b}\left(a I+\Gamma_{\eta}^{b}\right)^{-1} A R(b-\eta) R\left(\sigma_{n}\right) G\left(\eta, \bar{x}_{\eta}\right) d \eta \\
& -B^{*} R^{*}(b-s) \int_{0}^{b}\left(a I+\Gamma_{\eta}^{b}\right)^{-1} A R(b-\eta) \int_{0}^{\eta} h(\eta-v) R\left(\sigma_{n}\right) G\left(v, \bar{x}_{v}\right) d v d \eta \\
& -B^{*} R^{*}(b-s) \int_{0}^{b}\left(a I+\Gamma_{\eta}^{b}\right)^{-1} R(b-\eta) f(\eta) d w(\eta) \\
& \left.-B^{*} R^{*}(b-s)\left(a I+\Gamma_{\eta}^{b}\right)^{-1} \sum_{k=1}^{m} R\left(b-t_{k}\right) R\left(\sigma_{n}\right) I_{k}\left(\bar{x}_{t_{k}}\right)\right\} d s \\
& +\lambda \int_{0}^{t} R(t-s) f(s) d w(s)+\lambda \sum_{0<t_{k}<t} R\left(t-t_{k}\right) R\left(\sigma_{n}\right) I_{k}\left(\bar{x}_{t_{k}}\right), \quad t \in J
\end{aligned}
$$

for some $\lambda \in(0,1)$. On the other hand, from condition (H5), we conclude that there exist positive constants $\epsilon_{k}(k=1, \ldots, m), \gamma_{1}$ such that, for all $\|\psi\|_{\mathcal{B}}^{p}>\gamma_{1}$,

$$
\begin{aligned}
& E\left\|I_{k}(\psi)\right\|_{H}^{p} \leq\left(c_{k}+\epsilon_{k}\right)\|\psi\|_{\mathcal{B}}^{p}, \\
& 14^{p-1} K_{b}^{p} M^{p}\left[\left(\left\|(-A)^{-\beta}\right\|_{H}^{p}+M_{1-\beta}^{p} \frac{b^{p \beta}}{1-p(1-\beta)}\right.\right. \\
& \left.\left.\quad+M_{1-\beta}^{p} M_{1}^{p} \frac{b^{p(1+\beta)}}{1-p(1-\beta)}\right) L_{G}+m^{p-1} M^{p} \sum_{k=1}^{m}\left(c_{k}+\epsilon_{k}\right)\right] N_{*}^{p}<1 .
\end{aligned}
$$

Let

$$
F_{1}=\left\{\psi:\|\psi\|_{\mathcal{B}}^{p} \leq \gamma_{1}\right\}, \quad F_{2}=\left\{\psi:\|\psi\|_{\mathcal{B}}^{p}>\gamma_{1}\right\}, \quad C_{1}=\max \left\{E\left\|I_{k}(\psi)\right\|_{H}^{p}, \psi \in F_{1}\right\}
$$

Therefore

$$
E\left\|I_{k}(\psi)\right\|_{H}^{p} \leq C_{1}+\left(c_{k}+\epsilon_{k}\right)\|\psi\|_{\mathcal{B}}^{p}
$$

Then, by (H1)-(H4) and (3.7), from (3.5) we have for $t \in J$,

$$
\begin{aligned}
E\|x(t)\|_{H}^{p} \leq & 7^{p-1} E\left\|R(t)\left[\varphi(0)-R\left(\sigma_{n}\right) G(0, \varphi)\right]\right\|_{H}^{p}+7^{p-1} E\left\|R\left(\sigma_{n}\right) G\left(t, \bar{x}_{t}\right)\right\|_{H}^{p} \\
& +7^{p-1} E\left\|\int_{0}^{t} A R(t-s) R\left(\sigma_{n}\right) G\left(s, \bar{x}_{s}\right) d s\right\|_{H}^{p} \\
& +7^{p-1} E\left\|\int_{0}^{t} A R(t-s) \int_{0}^{s} h(s-\tau) R\left(\sigma_{n}\right) G\left(\tau, \bar{x}_{\tau}\right) d \tau d s\right\|_{H}^{p} \\
& +7^{p-1} E \| \int_{0}^{t} R(t-s) B\left\{B ^ { * } R ^ { * } ( b - s ) ( a I + \Gamma _ { 0 } ^ { b } ) ^ { - 1 } \left[E \tilde{x}_{b}+\int_{0}^{b} \tilde{\phi}(\eta) d w(\eta)\right.\right.
\end{aligned}
$$




$$
\begin{aligned}
& \left.-R(b)\left[\varphi(0)-R\left(\sigma_{n}\right) G(0, \varphi)\right]-R\left(\sigma_{n}\right) G\left(b, x_{b}\right)\right] \\
& -B^{*} R^{*}(b-s) \int_{0}^{b}\left(a I+\Gamma_{\eta}^{b}\right)^{-1} A R(b-\eta) R\left(\sigma_{n}\right) G\left(\eta, \bar{x}_{\eta}\right) d \eta \\
& -B^{*} R^{*}(b-s) \int_{0}^{b}\left(a I+\Gamma_{\eta}^{b}\right)^{-1} A R(b-\eta) \int_{0}^{\eta} h(\eta-v) R\left(\sigma_{n}\right) G\left(v, \bar{x}_{v}\right) d v d \eta \\
& -B^{*} R^{*}(b-s) \int_{0}^{b}\left(a I+\Gamma_{\eta}^{b}\right)^{-1} R(b-\eta) f(\eta) d w(\eta) \\
& \left.-B^{*} R^{*}(b-s)\left(a I+\Gamma_{\eta}^{b}\right)^{-1} \sum_{k=1}^{m} R\left(b-t_{k}\right) R\left(\sigma_{n}\right) I_{k}\left(\bar{x}_{t_{k}}\right)\right\} d s \|_{H}^{p} \\
& +7^{p-1} E\left\|\int_{0}^{t} R(t-s) f(s) d w(s)\right\|_{H}^{p}+7^{p-1} E\left\|\sum_{0<t_{k}<t} R\left(t-t_{k}\right) R\left(\sigma_{n}\right) I_{k}\left(\bar{x}_{t_{k}}\right)\right\|_{H}^{p} \\
& \leq 14^{p-1} M^{p} e^{-p \delta t}\left[\left(\tilde{H}\|\varphi\|_{\mathcal{B}}\right)^{p}+\left\|(-A)^{-\beta}\right\|_{H^{p}}^{p} M^{p} e^{-p \delta \sigma_{n}} L_{G}\left(\|\varphi\|_{\mathcal{B}}^{p}+1\right)\right] \\
& +7^{p-1}\left\|(-A)^{-\beta}\right\|_{H}^{p} M^{p} e^{-p \delta \sigma_{n}} L_{G}\left(\left\|\bar{x}_{t}\right\|_{\mathcal{B}}^{p}+1\right) \\
& +7^{p-1} M_{1-\beta}^{p} b^{p-1} \int_{0}^{t}(t-s)^{-p(1-\beta)} M^{p} e^{-p \delta \sigma_{n}} L_{G}\left(\left\|\bar{x}_{s}\right\|_{\mathcal{B}}^{p}+1\right) d s \\
& +7^{p-1} M_{1-\beta}^{p} b^{2(p-1)} M_{1}^{p} \int_{0}^{t}(t-s)^{-p(1-\beta)} \int_{0}^{s} M^{p} e^{-p \delta \sigma_{n}} L_{G}\left(\left\|\bar{x}_{\tau}\right\|_{\mathcal{B}}^{p}+1\right) d \tau d s \\
& +35^{p-1} M^{p} e^{-p \delta t} \frac{1}{a^{p}} M^{p} e^{-p \delta b} M_{2}^{2 p} b^{p-1} \int_{0}^{t} e^{2 p \delta s}\left\{4 ^ { p - 1 } \left[\left\|E \tilde{x}_{b}\right\|_{H}^{p}\right.\right. \\
& +C_{p} b^{p / 2-1} \int_{0}^{b} E\|\tilde{\phi}(\eta)\|_{H}^{p} d \eta+2^{p-1} M^{p} e^{-p \delta b}\left[E\|\varphi(0)\|_{H}^{p}\right. \\
& \left.+\left\|(-A)^{-\beta}\right\|_{H}^{p} M^{p} e^{-p \delta \sigma_{n}} L_{G}\left(\|\varphi\|_{\mathcal{B}}^{p}+1\right)\right] \\
& \left.+\left\|(-A)^{-\beta}\right\|_{H}^{p} M^{p} e^{-p \delta \sigma_{n}} L_{G}\left(\left\|\bar{x}_{b}\right\|_{\mathcal{B}}^{p}+1\right)\right] \\
& +M_{1-\beta}^{p} b^{p-1} \int_{0}^{b}(b-\eta)^{-p(1-\beta)} M^{p} e^{-p \delta \sigma_{n}} L_{G}\left(\left\|\bar{x}_{\eta}\right\|_{\mathcal{B}}^{p}+1\right) d s \\
& +M_{1-\beta}^{p} b^{2(p-1)} M_{1}^{p} \int_{0}^{b}(b-\eta)^{-p(1-\beta)} \int_{0}^{\eta} M^{p} e^{-p \delta \sigma_{n}} L_{G}\left(\left\|\bar{x}_{\nu}\right\|_{\mathcal{B}}^{p}+1\right) d \nu d \eta \\
& +C_{p} M^{p} b^{p / 2-1} e^{-p \delta b} \int_{0}^{b} e^{p \delta \eta} m_{f}(\eta) \Theta_{f}\left(\left\|\bar{x}_{\eta}\right\|_{\mathcal{B}}^{p}\right) d \eta \\
& \left.+M^{p} m^{p-1} \sum_{k=1}^{m} e^{-p \delta\left(b-t_{k}\right)} M^{p} e^{-p \delta \sigma_{n}}\left[C_{1}+\left(c_{k}+\epsilon_{k}\right)\left\|\bar{x}_{t_{k}}\right\|_{B}^{p}\right]\right\} d s \\
& +7^{p-1} C_{p} M^{p} b^{p / 2-1} e^{-p \delta t} \int_{0}^{t} e^{p \delta s} m_{f}(s) \Theta_{f}\left(\left\|\bar{x}_{s}\right\|_{\mathcal{B}}^{p}\right) d s \\
& +7^{p-1} M^{p} m^{p-1} \sum_{k=1}^{m} e^{-p \delta\left(t-t_{k}\right)} M^{p} e^{-p \delta \sigma_{n}}\left[C_{1}+\left(c_{k}+\epsilon_{k}\right)\left\|\bar{x}_{t_{k}}\right\|_{B}^{p}\right]
\end{aligned}
$$

where $M_{2}=\|B\|_{H}, C_{p}=(p(p-1) / 2)^{p / 2}$. By Lemma 2.3, it follows that

$$
\sup \left\{\left\|\bar{x}_{s}\right\|_{\mathcal{B}}^{p}: 0 \leq s \leq t\right\} \leq 2^{p-1}\left(M_{b} E\|\varphi\|_{\mathcal{B}}\right)^{p}+2^{p-1} K_{b}^{p} \sup \left\{E\|x(s)\|_{H}^{p}: 0 \leq s \leq t\right\}
$$


Consider the function defined by

$$
\zeta(t)=2^{p-1}\left(M_{b} E\|\varphi\|_{\mathcal{B}}\right)^{p}+2^{p-1} K_{b}^{p} \sup \left\{E\|x(s)\|_{H}^{p}: 0 \leq s \leq t\right\}, \quad 0 \leq t \leq b .
$$

For each $t \in[0, b]$, we have

$$
\begin{aligned}
\zeta(t) \leq & 2^{p-1}\left(M_{b} E\|\varphi\|_{\mathcal{B}}\right)^{p}+28^{p-1} K_{b}^{p} M^{p} e^{-p \delta t}\left[\left(\tilde{H}\|\varphi\|_{\mathcal{B}}\right)^{p}\right. \\
& \left.+\left\|(-A)^{-\beta}\right\|_{H}^{p} M^{p} e^{-p \delta \sigma_{n}} L_{G}\left(\|\varphi\|_{\mathcal{B}}^{p}+1\right)\right] \\
& +14^{p-1} K_{b}^{p}\left\|(-A)^{-\beta}\right\|_{H}^{p} M^{p} e^{-p \delta \sigma_{n}} L_{G}(\zeta(t)+1) \\
& +14^{p-1} K_{b}^{p} M_{1-\beta}^{p} \frac{b^{p \beta}}{1-p(1-\beta)} M^{p} e^{-p \delta \sigma_{n}} L_{G}(\zeta(t)+1) \\
& +14^{p-1} K_{b}^{p} M_{1-\beta}^{p} M_{1}^{p} \frac{b^{p(1+\beta)}}{1-p(1-\beta)} M^{p} e^{-p \delta \sigma_{n}} L_{G}(\zeta(t)+1) \\
& +e^{-p \delta t} \tilde{M}+70^{p-1} M^{p} e^{-p \delta t} \frac{1}{a^{p}} M^{p} e^{-p \delta b} M_{2}^{2 p} b^{p-1} K_{b}^{p} M^{p} m^{p-1} \\
& \times \sum_{k=1}^{m} e^{-p \delta\left(b-t_{k}\right)} M^{p} e^{-p \delta \sigma_{n}}\left(c_{k}+\epsilon_{k}\right) \int_{0}^{t} e^{2 p \delta s} \zeta(s) d s \\
& +14^{p-1} K_{b}^{p} C_{p} M^{p} b^{p / 2-1} e^{-p \delta t} \int_{0}^{t} e^{p \delta s} m_{f}(s) \Theta_{f}(\zeta(s)) d s \\
& +14^{p-1} K_{b}^{p} M^{p} m^{p-1} \sum_{k=1}^{m} e^{-p \delta\left(t-t_{k}\right)} M^{p} e^{-p \delta \sigma_{n}}\left(c_{k}+\epsilon_{k}\right) \zeta(t),
\end{aligned}
$$

where

$$
\begin{aligned}
\tilde{M}= & 70^{p-1} K_{b}^{p} \frac{1}{a^{p}} M^{p} e^{-p \delta b} M_{2}^{2 p} b^{p} N_{*}^{2 p}\left\{4 ^ { p - 1 } \left[\left\|E \tilde{x}_{b}\right\|_{H}^{p}+C_{p} b^{p / 2-1} \int_{0}^{b} E\|\tilde{\phi}(\eta)\|_{H}^{p} d \eta\right.\right. \\
& \left.+2^{p-1} M^{p} e^{-p \delta b}\left[E\|\varphi(0)\|_{H}^{p}+\left\|(-A)^{-\beta}\right\|_{H}^{p} M^{p} e^{-p \delta \sigma_{n}} L_{G}\left(\|\varphi\|_{\mathcal{B}}^{p}+1\right)\right]\right] \\
& +\left(4^{p-1}\left\|(-A)^{-\beta}\right\|_{H}^{p}+M_{1-\beta}^{p} \frac{b^{p \beta}}{1-p(1-\beta)}+M_{1-\beta}^{p} M_{1}^{p} \frac{b^{p(1+\beta)}}{1-p(1-\beta)}\right) \\
& \times M^{p} e^{-p \delta \sigma_{n}} L_{G}(\zeta(b)+1)+C_{p} M^{p} b^{p / 2-1} e^{-p \delta b} \int_{0}^{b} e^{p \delta \eta} m_{f}(\eta) \Theta_{f}(\zeta(\eta)) d \eta \\
& \left.+M^{p} m^{p} M^{p} e^{-p \delta b} N_{*}^{p} M^{p} e^{-p \delta \sigma_{n}} C_{1}\right\}+14^{p-1} K_{b}^{p} M^{p} m^{p} N_{*}^{p} M^{p} e^{-p \delta \sigma_{n}} C_{1} .
\end{aligned}
$$

Since $\lim _{n \rightarrow \infty} \sigma_{n}=0$, it follows that

$$
\begin{aligned}
\zeta(t) \leq & 2^{p-1}\left(M_{b} E\|\varphi\|_{\mathcal{B}}\right)^{p}+28^{p-1} K_{b}^{p} M^{p} e^{-p \delta t}\left[\left(\tilde{H}\|\varphi\|_{\mathcal{B}}\right)^{p}\right. \\
& \left.+\left\|(-A)^{-\beta}\right\|_{H^{p}}^{p} M^{p} L_{G}\left(\|\varphi\|_{\mathcal{B}}^{p}+1\right)\right] \\
& +14^{p-1} K_{b}^{p}\left\|(-A)^{-\beta}\right\|_{H^{p}}^{p} M^{p} L_{G}(\zeta(t)+1) \\
& +14^{p-1} K_{b}^{p} M_{1-\beta}^{p} \frac{b^{p \beta}}{1-p(1-\beta)} M^{p} L_{G}(\zeta(t)+1) \\
& +14^{p-1} K_{b}^{p} M_{1-\beta}^{p} M_{1}^{p} \frac{b^{p(1+\beta)}}{1-p(1-\beta)} M^{p} L_{G}(\zeta(t)+1)+e^{-p \delta t} \widetilde{M}
\end{aligned}
$$




$$
\begin{aligned}
& +\widetilde{M}_{1} e^{-p \delta t} \int_{0}^{t} e^{2 p \delta s} \zeta(s) d s+14^{p-1} K_{b}^{p} C_{p} M^{p} b^{p / 2-1} e^{-p \delta t} \int_{0}^{t} e^{p \delta s} m_{f}(s) \Theta_{f}(\zeta(s)) d s \\
& +14^{p-1} K_{b}^{p} M^{p} m^{p-1} e^{-p \delta t} N_{*}^{p} M^{p} \sum_{k=1}^{m}\left(c_{k}+\epsilon_{k}\right) \zeta(t),
\end{aligned}
$$

where $\widetilde{M}_{1}=70^{p-1} M^{p} \frac{1}{a^{p}} M^{p} e^{-p \delta b} M_{2}^{2 p} b^{p-1} K_{b}^{p} M^{p} m^{p-1} e^{-p \delta b} N_{*}^{p} M^{p} \sum_{k=1}^{m}\left(c_{k}+\epsilon_{k}\right)$. By using $\tilde{L}_{0}=$ $14^{p-1} K_{b}^{p} M^{p}\left[\left(\left\|(-A)^{-\beta}\right\|_{H}^{p}+M_{1-\beta}^{p} \frac{b^{p \beta}}{1-p(1-\beta)}+M_{1-\beta}^{p} M_{1}^{p} \frac{b^{p(1+\beta)}}{1-p(1-\beta)}\right) L_{G}+m^{p-1} M^{p} \sum_{k=1}^{m}\left(c_{k}+\epsilon_{k}\right)\right] N_{*}^{p}<$ 1 , we obtain that

$$
\begin{aligned}
e^{p \delta t} \zeta(t) \leq & \frac{1}{1-\tilde{L}_{0}}\left[\tilde{M}_{2}+\tilde{M}_{1} \int_{0}^{t} e^{2 p \delta s} \zeta(s) d s\right. \\
& \left.+14^{p-1} K_{b}^{p} C_{p} M^{p} b^{p / 2-1} \int_{0}^{t} e^{p \delta s} m_{f}(s) \Theta_{f}(\zeta(s)) d s\right]
\end{aligned}
$$

where

$$
\begin{aligned}
\widetilde{M}_{2}= & 2^{p-1} N_{*}^{p}\left(M_{b} E\|\varphi\|_{\mathcal{B}}\right)^{p}+28^{p-1} K_{b}^{p} M^{p}\left[\left(\tilde{H}\|\varphi\|_{\mathcal{B}}\right)^{p}\right. \\
& \left.+\left\|(-A)^{-\beta}\right\|_{H}^{p} M^{p} L_{G}\left(\|\varphi\|_{\mathcal{B}}^{p}+1\right)\right]+14^{p-1} K_{b}^{p} M^{p} N_{*}^{p}\left[\left\|(-A)^{-\beta}\right\|_{H}^{p}\right. \\
& \left.+M_{1-\beta}^{p} \frac{b^{p \beta}}{1-p(1-\beta)}+M_{1-\beta}^{p} M_{1}^{p} \frac{b^{p(1+\beta)}}{1-p(1-\beta)}\right] L_{G}+\widetilde{M} .
\end{aligned}
$$

Denoting by $v(t)$ the right-hand side of the above inequality, we get that $\zeta(t) \leq e^{-p \delta t} v(t)$ for all $t \in J$, and $v(0)=\frac{1}{1-\tilde{L}_{0}} \widetilde{M}_{2}$,

$$
\begin{aligned}
v^{\prime}(t) & =\frac{1}{1-\tilde{L}_{0}}\left[\tilde{M}_{1} e^{2 p \delta t} \zeta(t)+14^{p-1} K_{b}^{p} C_{p} M^{p} b^{p / 2-1} e^{p \delta t} m_{f}(t) \Theta_{f}(\zeta(t))\right] \\
& \leq \frac{1}{1-\tilde{L}_{0}}\left[\tilde{M}_{1} e^{2 p \delta t} e^{-p \delta t} v(t)+14^{p-1} K_{b}^{p} C_{p} M^{p} b^{p / 2-1} e^{p \delta t} m_{f}(t) \Theta_{f}\left(e^{-p \delta t} v(t)\right)\right], \quad t \in J .
\end{aligned}
$$

Then, for each $t \in J$, we have

$$
\begin{aligned}
\left(e^{-p \delta t} v(t)\right)^{\prime}= & (-p \delta) e^{-p \delta t} v(t)+v^{\prime}(t) e^{-p \delta t} \\
\leq & (-p \delta) e^{-p \delta t} v(t)+\frac{1}{1-\tilde{L}_{0}} \widetilde{M}_{1} v(t) \\
& +\frac{1}{1-\tilde{L}_{0}} 14^{p-1} K_{b}^{p} C_{p} M^{p} b^{p / 2-1} m_{f}(t) \Theta_{f}\left(e^{-p \delta t} v(t)\right) \\
\leq & \max \left\{(-p \delta)+\frac{1}{1-\tilde{L}_{0}} \widetilde{M}_{1} e^{p \delta t}, \frac{1}{1-\tilde{L}_{0}} 14^{p-1} K_{b}^{p} C_{p} M^{p} b^{p / 2-1} m_{f}(t)\right\} \\
& \times\left[e^{-p \delta t} v(t)+\Theta_{f}\left(e^{-p \delta t} v(t)\right)\right], \quad t \in J .
\end{aligned}
$$

This implies that

$$
\begin{aligned}
\int_{v(0)}^{e^{-p \delta t} v(t)} \frac{d \vartheta}{\vartheta+\Theta_{f}(\vartheta)} \leq & \int_{0}^{b} \max \left\{(-p \delta)+\frac{1}{1-\tilde{L}_{0}} \widetilde{M}_{1} e^{p \delta t}\right. \\
& \left.\frac{1}{1-\tilde{L}_{0}} 14^{p-1} K_{b}^{p} C_{p} M^{p} b^{p / 2-1} m_{f}(t)\right\} d t<\infty
\end{aligned}
$$


This inequality shows that there is a constant $\widetilde{K}$ such that $e^{-p \delta t} v(t) \leq \widetilde{K}, t \in J$, and hence $\|x\|_{\mathcal{P C}}^{p} \leq e^{-p \delta t} v(t) \leq \widetilde{K}$, where $\widetilde{K}$ depends only on $M, \delta, p, C_{p}, K_{b}, b$ and on the functions $m_{f}(\cdot)$, and $\Theta_{f}(\cdot)$. Then there exists $r$ such that $\|x\|_{\mathcal{P C}}^{p} \neq r^{*}$. Set

$$
V=\left\{x \in \mathcal{B P C}:\|x\|_{\mathcal{P C}}^{p}<r^{*}\right\}
$$

From the choice of $V$, there is no $x \in \partial V$ such that $x \in \lambda \Phi_{n} x$ for $\lambda \in(0,1)$.

Step 2. $\Phi_{n}$ has a closed graph.

Let $x^{(j)} \rightarrow x^{*}(n \rightarrow \infty), \rho_{n}^{(j)} \in \Phi_{n} x^{(j)}, x^{(j)} \in \bar{V}=B_{r^{*}}(0, \mathcal{B P C})$ and $\rho_{n}^{(j)} \rightarrow \rho_{n}^{*}$. From axiom (A), it is easy to see that $\left(\overline{x^{(j)}}\right)_{s} \rightarrow\left(\overline{x^{*}}\right)_{s}$ uniformly for $s \in(-\infty, b]$ as $j \rightarrow \infty$. We shall prove that $\rho_{n}^{*} \in \Phi_{n} \overline{x^{*}}$. Now $\rho_{n}^{(j)} \in \Phi_{n} \overline{x^{(j)}}$ means that there exists $f^{(j)} \in S_{F, x^{(j)}}$ such that, for each $t \in J$,

$$
\begin{aligned}
\rho_{n}^{(j)}(t)= & R(t)\left[\varphi(0)-R\left(\sigma_{n}\right) G(0, \varphi)\right]+R\left(\sigma_{n}\right) G\left(t,\left(\overline{x^{(j)}}\right)_{t}\right) \\
& +\int_{0}^{t} A R(t-s) R\left(\sigma_{n}\right) G\left(s,\left(\overline{x^{(j)}}\right)_{s}\right) d s \\
& +\int_{0}^{t} A R(t-s) \int_{0}^{s} h(s-\tau) R\left(\sigma_{n}\right) G\left(\tau,\left(\overline{x^{(j)}}\right)_{\tau}\right) d \tau d s \\
& +\int_{0}^{t} R(t-s) B\left\{B ^ { * } R ^ { * } ( b - s ) ( a I + \Gamma _ { 0 } ^ { b } ) ^ { - 1 } \left[E \tilde{x}_{b}+\int_{0}^{b} \tilde{\phi}(\eta) d w(\eta)\right.\right. \\
& \left.-R(b)\left[\varphi(0)-R\left(\sigma_{n}\right) G(0, \varphi)\right]-R\left(\sigma_{n}\right) G\left(b,\left(\overline{x^{(j)}}\right)_{b}\right)\right] \\
& -B^{*} R^{*}(b-s) \int_{0}^{b}\left(a I+\Gamma_{\eta}^{b}\right)^{-1} A R(b-\eta) R\left(\sigma_{n}\right) G\left(\eta,\left(\overline{x^{(j)}}\right)_{\eta}\right) d \eta \\
& \left.-B^{*} R^{*}(b-s) \int_{0}^{b}\left(a I+\Gamma_{\eta}^{b}\right)^{-1} A R(b-\eta) \int_{0}^{\eta} h(\eta-v) R\left(\sigma_{n}\right) G\left(v, \overline{\left.x^{(j)}\right)}\right)_{v}\right) d v d \eta \\
& -B^{*} R^{*}(b-s) \int_{0}^{b}\left(a I+\Gamma_{\eta}^{b}\right)^{-1} R(b-\eta) f^{(j)}(\eta) d w(\eta) \\
& \left.-B^{*} R^{*}(b-s)\left(a I+\Gamma_{\eta}^{b}\right)^{-1} \sum_{k=1}^{m} R\left(b-t_{k}\right) R\left(\sigma_{n}\right) I_{k}\left(\left(\overline{x^{(j)}}\right)_{t_{k}}\right)\right\} d s \\
& +\int_{0}^{t} R(t-s) f^{(j)}(s) d w(s)+\sum_{0<t_{k}<t} R\left(t-t_{k}\right) R\left(\sigma_{n}\right) I_{k}\left(\left(\overline{x^{(j)}}\right)_{t_{k}}\right), \quad t \in J .
\end{aligned}
$$

We must prove that there exists $f^{*} \in S_{F, \overline{x^{*}}}$ such that, for each $t \in J$,

$$
\begin{aligned}
\rho_{n}^{*}(t)= & R(t)\left[\varphi(0)-R\left(\sigma_{n}\right) G(0, \varphi)\right]+R\left(\sigma_{n}\right) G\left(t,\left(\overline{x^{*}}\right)_{t}\right)+\int_{0}^{t} A R(t-s) R\left(\sigma_{n}\right) G\left(s,\left(\overline{x^{*}}\right)_{s}\right) d s \\
& +\int_{0}^{t} A R(t-s) \int_{0}^{s} h(s-\tau) R\left(\sigma_{n}\right) G\left(\tau,\left(\overline{x^{*}}\right)_{\tau}\right) d \tau d s \\
& +\int_{0}^{t} R(t-s) B\left\{B ^ { * } R ^ { * } ( b - s ) ( a I + \Gamma _ { 0 } ^ { b } ) ^ { - 1 } \left[E \tilde{x}_{b}+\int_{0}^{b} \tilde{\phi}(\eta) d w(\eta)\right.\right. \\
& \left.-R(b)\left[\varphi(0)-R\left(\sigma_{n}\right) G(0, \varphi)\right]-R\left(\sigma_{n}\right) G\left(b,\left(\overline{x^{*}}\right)_{b}\right)\right]
\end{aligned}
$$


Pan and Lu Advances in Difference Equations (2015) 2015:106

Page 16 of 34

$$
\begin{aligned}
& -B^{*} R^{*}(b-s) \int_{0}^{b}\left(a I+\Gamma_{\eta}^{b}\right)^{-1} A R(b-\eta) R\left(\sigma_{n}\right) G\left(\eta,\left(\overline{x^{*}}\right)_{\eta}\right) d \eta \\
& -B^{*} R^{*}(b-s) \int_{0}^{b}\left(a I+\Gamma_{\eta}^{b}\right)^{-1} A R(b-\eta) \int_{0}^{\eta} h(\eta-v) R\left(\sigma_{n}\right) G\left(v,\left(\overline{x^{*}}\right)_{v}\right) d v d \eta \\
& -B^{*} R^{*}(b-s) \int_{0}^{b}\left(a I+\Gamma_{\eta}^{b}\right)^{-1} R(b-\eta) f^{*}(\eta) d w(\eta) \\
& \left.-B^{*} R^{*}(b-s)\left(a I+\Gamma_{\eta}^{b}\right)^{-1} \sum_{k=1}^{m} R\left(b-t_{k}\right) R\left(\sigma_{n}\right) I_{k}\left(\left(\overline{x^{*}}\right)_{t_{k}}\right)\right\} d s \\
& +\int_{0}^{t} R(t-s) f^{*}(s) d w(s)+\sum_{0<t_{k}<t} R\left(t-t_{k}\right) R\left(\sigma_{n}\right) I_{k}\left(\left(\overline{x^{*}}\right)_{t_{k}}\right), \quad t \in J .
\end{aligned}
$$

Now, for every $t \in J$, we have

$$
\begin{aligned}
& \|\left(\rho_{n}^{(j)}(t)-R(t)\left[\varphi(0)-R\left(\sigma_{n}\right) G(0, \varphi)\right]-R\left(\sigma_{n}\right) G\left(t,\left(\overline{x^{(j)}}\right)_{t}\right)\right. \\
& -\int_{0}^{t} A R(t-s) R\left(\sigma_{n}\right) G\left(s,\left(\overline{x^{(j)}}\right)_{s}\right) d s \\
& -\int_{0}^{t} A R(t-s) \int_{0}^{s} h(s-\tau) R\left(\sigma_{n}\right) G\left(\tau,\left(\overline{x^{(j)}}\right)_{\tau}\right) d \tau d s \\
& -\int_{0}^{t} R(t-s) B\left\{B ^ { * } R ^ { * } ( b - s ) ( a I + \Gamma _ { 0 } ^ { b } ) ^ { - 1 } \left[E \tilde{x}_{b}+\int_{0}^{b} \tilde{\phi}(\eta) d w(\eta)\right.\right. \\
& \left.-R(b)\left[\varphi(0)-R\left(\sigma_{n}\right) G(0, \varphi)\right]-R\left(\sigma_{n}\right) G\left(b,\left(\overline{x^{(j)}}\right)_{b}\right)\right] \\
& -B^{*} R^{*}(b-s) \int_{0}^{b}\left(a I+\Gamma_{\eta}^{b}\right)^{-1} A R(b-\eta) R\left(\sigma_{n}\right) G\left(\eta,\left(\overline{x^{(j)}}\right)_{\eta}\right) d \eta \\
& -B^{*} R^{*}(b-s) \int_{0}^{b}\left(a I+\Gamma_{\eta}^{b}\right)^{-1} A R(b-\eta) \int_{0}^{\eta} h(\eta-v) R\left(\sigma_{n}\right) G\left(v,\left(\overline{x^{(j)}}\right)_{v}\right) d v d \eta \\
& \left.-B^{*} R^{*}(b-s)\left(a I+\Gamma_{\eta}^{b}\right)^{-1} \sum_{k=1}^{m} R\left(b-t_{k}\right) R\left(\sigma_{n}\right) I_{k}\left(\left(\overline{x^{(j)}}\right)_{t_{k}}\right)\right\} d s \\
& \left.-\sum_{0<t_{k}<t} R\left(t-t_{k}\right) R\left(\sigma_{n}\right) I_{k}\left(\left(\overline{x^{(j)}}\right)_{t_{k}}\right)\right) \\
& -\left(\rho_{n}^{*}(t)-R(t)\left[\varphi(0)-R\left(\sigma_{n}\right) G(0, \varphi)\right]-R\left(\sigma_{n}\right) G\left(t,\left(\overline{x^{*}}\right)_{t}\right)\right. \\
& -\int_{0}^{t} A R(t-s) R\left(\sigma_{n}\right) G\left(s,\left(\overline{x^{*}}\right)_{s}\right) d s \\
& -\int_{0}^{t} A R(t-s) \int_{0}^{s} h(s-\tau) R\left(\sigma_{n}\right) G\left(\tau,\left(\overline{x^{*}}\right)_{\tau}\right) d \tau d s \\
& -\int_{0}^{t} R(t-s) B\left\{B ^ { * } R ^ { * } ( b - s ) ( a I + \Gamma _ { 0 } ^ { b } ) ^ { - 1 } \left[E \tilde{x}_{b}+\int_{0}^{b} \tilde{\phi}(\eta) d w(\eta)\right.\right. \\
& \left.-R(b)\left[\varphi(0)-R\left(\sigma_{n}\right) G(0, \varphi)\right]-R\left(\sigma_{n}\right) G\left(b,\left(\overline{x^{*}}\right)_{b}\right)\right]
\end{aligned}
$$




$$
\begin{aligned}
& -B^{*} R^{*}(b-s) \int_{0}^{b}\left(a I+\Gamma_{\eta}^{b}\right)^{-1} A R(b-\eta) R\left(\sigma_{n}\right) G\left(\eta,\left(\overline{x^{*}}\right)_{\eta}\right) d \eta \\
& -B^{*} R^{*}(b-s) \int_{0}^{b}\left(a I+\Gamma_{\eta}^{b}\right)^{-1} A R(b-\eta) \int_{0}^{\eta} h(\eta-v) R\left(\sigma_{n}\right) G\left(v,\left(\overline{x^{*}}\right)_{v}\right) d v d \eta \\
& \left.-B^{*} R^{*}(b-s)\left(a I+\Gamma_{\eta}^{b}\right)^{-1} \sum_{k=1}^{m} R\left(b-t_{k}\right) R\left(\sigma_{n}\right) I_{k}\left(\left(\overline{x^{*}}\right)_{t_{k}}\right)\right\} d s \\
& \left.-\sum_{0<t_{k}<t} R\left(t-t_{k}\right) R\left(\sigma_{n}\right) I_{k}\left(\left(\overline{x^{*}}\right)_{t_{k}}\right)\right) \|_{\mathcal{P C}}^{p} \rightarrow 0 \quad \text { as } j \rightarrow \infty .
\end{aligned}
$$

Consider the linear continuous operator $\Psi: L^{p}(J, H) \rightarrow C(J, H)$,

$$
\begin{aligned}
\Psi(f)(t)= & \int_{0}^{t} R(t-s) f(s) d w(s) \\
& +\int_{0}^{t} R(t-s) B B^{*} R^{*}(b-s)\left(\int_{0}^{b}\left(a I+\Gamma_{\eta}^{b}\right)^{-1} R(b-\eta) f(\eta) d w(\eta)\right) d s
\end{aligned}
$$

From Lemma 3.1 it follows that $\Psi \circ S_{F}$ is a closed graph operator. Also, from the definition of $\Psi$, we have that, for every $t \in J$,

$$
\begin{aligned}
\rho_{n}^{(j)} & (t)-R(t)\left[\varphi(0)-R\left(\sigma_{n}\right) G(0, \varphi)\right]-R\left(\sigma_{n}\right) G\left(t,\left(\overline{x^{(j)}}\right)_{t}\right) \\
& -\int_{0}^{t} A R(t-s) R\left(\sigma_{n}\right) G\left(s,\left(\overline{x^{(j)}}\right)_{s}\right) d s \\
& -\int_{0}^{t} A R(t-s) \int_{0}^{s} h(s-\tau) R\left(\sigma_{n}\right) G\left(\tau,\left(\overline{x^{(j)}}\right)_{\tau}\right) d \tau d s \\
& -\int_{0}^{t} R(t-s) B\left\{B ^ { * } R ^ { * } ( b - s ) ( a I + \Gamma _ { 0 } ^ { b } ) ^ { - 1 } \left[E \tilde{x}_{b}+\int_{0}^{b} \tilde{\phi}(\eta) d w(\eta)\right.\right. \\
& \left.-R(b)\left[\varphi(0)-R\left(\sigma_{n}\right) G(0, \varphi)\right]-R\left(\sigma_{n}\right) G\left(b,\left(\overline{x^{(j)}}\right)_{b}\right)\right] \\
& -B^{*} R^{*}(b-s) \int_{0}^{b}\left(a I+\Gamma_{\eta}^{b}\right)^{-1} A R(b-\eta) R\left(\sigma_{n}\right) G\left(\eta,\left(\overline{x^{(j)}}\right)_{\eta}\right) d \eta \\
& -B^{*} R^{*}(b-s) \int_{0}^{b}\left(a I+\Gamma_{\eta}^{b}\right)^{-1} A R(b-\eta) \int_{0}^{\eta} h(\eta-v) R\left(\sigma_{n}\right) G\left(v,\left(\overline{x^{(j)}}\right)_{v}\right) d v d \eta \\
& \left.-B^{*} R^{*}(b-s)\left(a I+\Gamma_{\eta}^{b}\right)^{-1} \sum_{k=1}^{m} R\left(b-t_{k}\right) R\left(\sigma_{n}\right) I_{k}\left(\left(\overline{x^{(j)}}\right)_{t_{k}}\right)\right\} d s \\
& -\sum_{0<t_{k}<t} R\left(t-t_{k}\right) R\left(\sigma_{n}\right) I_{k}\left(\left(\overline{x^{(j)}}\right)_{t_{k}}\right) \in \Psi\left(S_{F, \overline{x(j)}}\right) .
\end{aligned}
$$

Since $\overline{x^{(j)}} \rightarrow \overline{x^{*}}$, for some $f^{*} \in S_{F, \overline{x^{*}}}$ it follows that, for every $t \in J$, we have

$$
\begin{aligned}
\rho_{n}^{*}(t) & -R(t)\left[\varphi(0)-R\left(\sigma_{n}\right) G(0, \varphi)\right]-R\left(\sigma_{n}\right) G\left(t,\left(\overline{x^{*}}\right)_{t}\right) \\
& -\int_{0}^{t} A R(t-s) R\left(\sigma_{n}\right) G\left(s,\left(\overline{x^{*}}\right)_{s}\right) d s
\end{aligned}
$$




$$
\begin{aligned}
& -\int_{0}^{t} A R(t-s) \int_{0}^{s} h(s-\tau) R\left(\sigma_{n}\right) G\left(\tau,\left(\overline{x^{*}}\right)_{\tau}\right) d \tau d s \\
& -\int_{0}^{t} R(t-s) B\left\{B ^ { * } R ^ { * } ( b - s ) ( a I + \Gamma _ { 0 } ^ { b } ) ^ { - 1 } \left[E \tilde{x}_{b}+\int_{0}^{b} \tilde{\phi}(\eta) d w(\eta)\right.\right. \\
& \left.\left.-R(b)\left[\varphi(0)-R\left(\sigma_{n}\right) G(0, \varphi)\right]-R\left(\sigma_{n}\right) G\left(b, \overline{x^{*}}\right)_{b}\right)\right] \\
& -B^{*} R^{*}(b-s) \int_{0}^{b}\left(a I+\Gamma_{\eta}^{b}\right)^{-1} A R(b-\eta) R\left(\sigma_{n}\right) G\left(\eta,\left(\overline{x^{*}}\right)_{\eta}\right) d \eta \\
& -B^{*} R^{*}(b-s) \int_{0}^{b}\left(a I+\Gamma_{\eta}^{b}\right)^{-1} A R(b-\eta) \int_{0}^{\eta} h(\eta-v) R\left(\sigma_{n}\right) G\left(v,\left(\overline{x^{*}}\right)_{v}\right) d v d \eta \\
& \left.-B^{*} R^{*}(b-s)\left(a I+\Gamma_{\eta}^{b}\right)^{-1} \sum_{k=1}^{m} R\left(b-t_{k}\right) R\left(\sigma_{n}\right) I_{k}\left(\left(\overline{x^{*}}\right)_{t_{k}}\right)\right\} d s \\
& \quad-\sum_{0<t_{k}<t} R\left(t-t_{k}\right) R\left(\sigma_{n}\right) I_{k}\left(\left(\overline{x^{*}}\right)_{t_{k}}\right) \\
& =\int_{0}^{t} R(t-s) f^{*}(s) d w(s) \\
& \quad+\int_{0}^{t} R(t-s) B B^{*} R^{*}(b-s)\left(\int_{0}^{b}\left(a I+\Gamma_{\eta}^{b}\right)^{-1} R(b-\eta) f^{*}(\eta) d w(\eta)\right) d s .
\end{aligned}
$$

Therefore, $\Phi_{n}$ has a closed graph.

Step 3. We show that the operator $\Phi_{n}$ is condensing.

For this purpose, we decompose $\Phi_{n}$ as $\Phi_{n}^{1}+\Phi_{n}^{2}$, where the map $\Phi_{n}^{1}: \bar{V} \rightarrow \mathcal{B P C}$ is defined by $\Phi_{n}^{1} x$, the set $\rho_{n}^{1} \in \mathcal{B P C}$ is such that

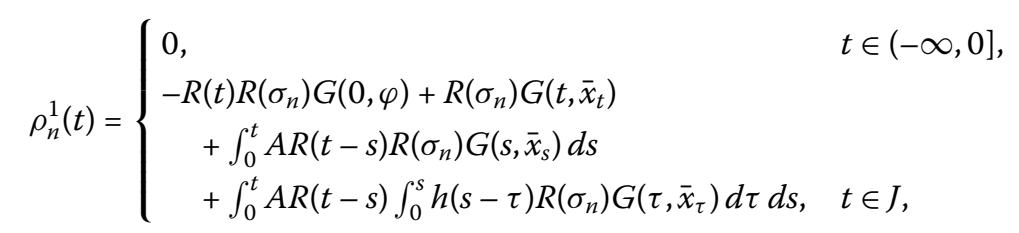

and the map $\Phi_{n}^{2}: \bar{V} \rightarrow \mathcal{B P C}$ is defined by $\Phi_{n}^{2} x$, the set $\rho_{n}^{2} \in \mathcal{B P C}$ is such that

$$
\rho_{n}^{2}(t)= \begin{cases}0, & t \in(-\infty, 0] \\ R(t) \varphi(0)+\int_{0}^{t} R(t-s) B u_{\bar{x}}^{a}(s) d s+\int_{0}^{t} R(t-s) f(s) d w(s) & \\ \quad+\sum_{0<t_{k}<t} R\left(t-t_{k}\right) R\left(\sigma_{n}\right) I_{k}\left(\bar{x}_{t_{k}}\right), & t \in J .\end{cases}
$$

We first show that $\Phi_{n}^{1}$ is a contraction while $\Phi_{n}^{2}$ is a completely continuous operator. (i) $\Phi_{n}^{1}$ is a contraction on $\bar{V}$.

Let $t \in J$ and $x^{*}, x^{* *} \in \mathcal{B P C}$. By using (H4), Hölder's inequality and Lemma 2.3, we have

$$
\begin{aligned}
E \| & \left(\Phi_{n}^{1} x^{*}\right)(t)-\left(\Phi_{n}^{1} x^{* *}\right)(t) \|_{H}^{p} \\
\leq & 3^{p-1} E\left\|(-A)^{-\beta}\right\|_{H}^{p}\left\|R\left(\sigma_{n}\right)\left[(-A)^{\beta} G\left(t, \overline{x^{*}} t\right)-(-A)^{\beta} G\left(t, \overline{x^{* *}} t\right)\right]\right\|_{H}^{p} \\
& \quad+3^{p-1} E\left\|\int_{0}^{t}(-A)^{1-\beta} R(t-s) R\left(\sigma_{n}\right)\left[(-A)^{\beta} G\left(s, \overline{x^{*}} s\right)-(-A)^{\beta} G\left(s, \overline{x^{* *}} s\right)\right] d s\right\|_{H}^{p}
\end{aligned}
$$




$$
\begin{aligned}
& +3^{p-1} E \| \int_{0}^{t}(-A)^{1-\beta} R(t-s) \int_{0}^{s} h(s-\tau) R\left(\sigma_{n}\right) \\
& \times\left[(-A)^{\beta} G\left(\tau, \overline{x^{*}}{ }_{\tau}\right)-(-A)^{\beta} G\left(\tau, \overline{x^{* *}}{ }_{\tau}\right)\right] d \tau d s \|_{H}^{p} \\
& \leq 3^{p-1}\left\|(-A)^{-\beta}\right\|_{H}^{p} L_{G} M^{p} e^{-p \delta \sigma_{n}}\left\|\overline{x^{*}} t-\overline{x^{* *}}\right\|_{\mathcal{B}}^{p} \\
& +3^{p-1} M_{1-\beta}^{p} b^{p-1} \int_{0}^{t}(t-s)^{-p(1-\beta)} L_{G} M^{p} e^{-p \delta \sigma_{n}}\left\|\overline{x^{*}}{ }_{s}-\overline{x^{* *}}{ }_{s}\right\|_{\mathcal{B}}^{p} d s \\
& +3^{p-1} M_{1-\beta}^{p} M_{1}^{p} b^{2(p-1)} \int_{0}^{t}(t-s)^{-p(1-\beta)} \int_{0}^{s} L_{G} M^{p} e^{-p \delta \sigma_{n}}\left\|\overline{x^{*}}{ }_{\tau}-\overline{x^{* *}}{ }_{\tau}\right\|_{\mathcal{B}}^{p} d \tau d s \\
& \leq 6^{p-1}\left\|(-A)^{-\beta}\right\|_{H}^{p} K_{b}^{p} L_{G} M^{p} e^{-p \delta \sigma_{n}} \sup _{s \in[0, b]} E\left\|\overline{x^{*}}(s)-\overline{x^{* *}}(s)\right\|_{H}^{p} \\
& +6^{p-1} M_{1-\beta}^{p} K_{b}^{p} L_{G} M^{p} e^{-p \delta \sigma_{n}} b^{p-1} \int_{0}^{t}(t-s)^{-p(1-\beta)} d s \sup _{s \in[0, b]} E\left\|\overline{x^{*}}(s)-\overline{x^{* *}}(s)\right\|_{H}^{p} \\
& +6^{p-1} M_{1-\beta}^{p} M_{1}^{p} b^{2(p-1)} K_{b}^{p} L_{G} M^{p} e^{-p \delta \sigma_{n}} b \int_{0}^{t}(t-s)^{-p(1-\beta)} d s \\
& \times \sup _{s \in[0, b]} E\left\|\overline{x^{*}}(s)-\overline{x^{* *}}(s)\right\|_{H}^{p} \\
& \leq 6^{p-1} K_{b}^{p} L_{G} M^{p} e^{-p \delta \sigma_{n}}\left[\left\|(-A)^{-\beta}\right\|_{H}^{p}+M_{1-\beta}^{p} \frac{b^{p \beta}}{1-p(1-\beta)}\right. \\
& \left.+M_{1-\beta}^{p} M_{1}^{p} \frac{b^{p(1+\beta)}}{1-p(1-\beta)}\right] \sup _{s \in[0, b]} E\left\|x^{*}(s)-x^{* *}(s)\right\|_{H}^{p} \quad(\text { since } \bar{x}=x \text { on } J) \\
& =6^{p-1} K_{b}^{p} L_{G} M^{p} e^{-p \delta \sigma_{n}}\left[\left\|(-A)^{-\beta}\right\|_{H}^{p}+M_{1-\beta}^{p} \frac{b^{p \beta}}{1-p(1-\beta)}\right. \\
& \left.+M_{1-\beta}^{p} M_{1}^{p} \frac{b^{p(1+\beta)}}{1-p(1-\beta)}\right]\left\|x^{*}-x^{* *}\right\|_{\mathcal{P C}}^{p} .
\end{aligned}
$$

Since $\lim _{n \rightarrow \infty} \sigma_{n}=0$, it follows that

$$
\left\|\left(\Phi_{n}^{1} x^{*}\right)(t)-\left(\Phi_{n}^{1} x^{* *}\right)(t)\right\|_{\mathcal{P C}}^{p} \leq L_{0}\left\|x^{*}-x^{* *}\right\|_{\mathcal{P C}}^{p} .
$$

Taking supremum over $t$, we have for all $t \in J$,

$$
\left\|\Phi_{n}^{1} x^{*}-\Phi_{n}^{1} x^{* *}\right\|_{\mathcal{P C}}^{p} \leq L_{0}\left\|x^{*}-x^{* *}\right\|_{\mathcal{P C}}^{p}
$$

where $L_{0}=6^{p-1} K_{b}^{p} L_{G} M^{p}\left[\left\|(-A)^{-\beta}\right\|_{H}^{p}+M_{1-\beta}^{p} \frac{b^{p \beta}}{1-p(1-\beta)}+M_{1-\beta}^{p} M_{1}^{p} \frac{b^{p(1+\beta)}}{1-p(1-\beta)}\right]$. By (3.1), we see that $L_{0}<1$. Hence, $\Phi_{n}^{1}$ is a contraction on $\mathcal{B P C}$.

(ii) $\Phi_{n}^{2}$ is convex for each $x \in \bar{V}$.

In fact, if $\tilde{\rho}_{n}^{1}, \tilde{\rho}_{n}^{2}$ belong to $\Phi_{n}^{2} x$, then there exist $f_{1}, f_{2} \in S_{F, \bar{x}}$ such that

$$
\begin{aligned}
\tilde{\rho}_{n}^{i}(t)= & R(t) \varphi(0)+\int_{0}^{t} R(t-s) B\left(B ^ { * } R ^ { * } ( b - s ) ( a I + \Gamma _ { 0 } ^ { b } ) ^ { - 1 } \left[E \tilde{x}_{b}+\int_{0}^{b} \tilde{\phi}(\eta) d w(\eta)\right.\right. \\
& \left.-R(b)\left[\varphi(0)-R\left(\sigma_{n}\right) G(0, \varphi)\right]-R\left(\sigma_{n}\right) G\left(b, x_{b}\right)\right] \\
& -B^{*} R^{*}(b-s) \int_{0}^{b}\left(a I+\Gamma_{\eta}^{b}\right)^{-1} A R(b-\eta) R\left(\sigma_{n}\right) G\left(\eta, \bar{x}_{\eta}\right) d \eta
\end{aligned}
$$




$$
\begin{aligned}
& -B^{*} R^{*}(b-s) \int_{0}^{b}\left(a I+\Gamma_{\eta}^{b}\right)^{-1} A R(b-\eta) \int_{0}^{\eta} h(\eta-v) R\left(\sigma_{n}\right) G\left(v, \bar{x}_{v}\right) d v d \eta \\
& -B^{*} R^{*}(b-s) \int_{0}^{b}\left(a I+\Gamma_{\eta}^{b}\right)^{-1} R(b-\eta) f_{i}(\eta) d w(\eta) \\
& \left.-B^{*} R^{*}(b-s)\left(a I+\Gamma_{\eta}^{b}\right)^{-1} \sum_{k=1}^{m} R\left(b-t_{k}\right) R\left(\sigma_{n}\right) I_{k}\left(\bar{x}_{t_{k}}\right)\right\} d s \\
& +\int_{0}^{t} R(t-s) f_{i}(s) d w(s)+\sum_{0<t_{k}<t} R\left(t-t_{k}\right) R\left(\sigma_{n}\right) I_{k}\left(\bar{x}_{t_{k}}\right), \quad t \in J, i=1,2 .
\end{aligned}
$$

Let $0 \leq \lambda \leq 1$. For each $t \in J$, we have

$$
\begin{aligned}
\left(\lambda \tilde{\rho}_{n}^{1}+\right. & \left.(1-\lambda) \tilde{\rho}_{n}^{2}\right)(t) \\
= & R(t) \varphi(0)+\int_{0}^{t} R(t-s) B\left\{B ^ { * } R ^ { * } ( b - s ) ( a I + \Gamma _ { 0 } ^ { b } ) ^ { - 1 } \left[E \tilde{x}_{b}+\int_{0}^{b} \tilde{\phi}(\eta) d w(\eta)\right.\right. \\
& \left.-R(b)\left[\varphi(0)-R\left(\sigma_{n}\right) G(0, \varphi)\right]-R\left(\sigma_{n}\right) G\left(b, x_{b}\right)\right] \\
& -B^{*} R^{*}(b-s) \int_{0}^{b}\left(a I+\Gamma_{\eta}^{b}\right)^{-1} A R(b-\eta) R\left(\sigma_{n}\right) G\left(\eta, \bar{x}_{\eta}\right) d \eta \\
& -B^{*} R^{*}(b-s) \int_{0}^{b}\left(a I+\Gamma_{\eta}^{b}\right)^{-1} A R(b-\eta) \int_{0}^{\eta} h(\eta-v) R\left(\sigma_{n}\right) G\left(v, \bar{x}_{v}\right) d v d \eta \\
& -B^{*} R^{*}(b-s) \int_{0}^{b}\left(a I+\Gamma_{\eta}^{b}\right)^{-1} R(b-\eta)\left[\lambda f_{1}(\eta)+(1-\lambda) f_{2}(\eta)\right] d w(\eta) \\
& \left.-B^{*} R^{*}(b-s)\left(a I+\Gamma_{\eta}^{b}\right)^{-1} \sum_{k=1}^{m} R\left(b-t_{k}\right) R\left(\sigma_{n}\right) I_{k}\left(\bar{x}_{t_{k}}\right)\right\} d s \\
& +\int_{0}^{t} R(t-s)\left[\lambda f_{1}(s)+(1-\lambda) f_{2}(s)\right] d w(s) \\
& +\sum_{0<t_{k}<t} R\left(t-t_{k}\right) R\left(\sigma_{n}\right) I_{k}\left(\bar{x}_{t_{k}}\right) .
\end{aligned}
$$

Since $S_{F, \bar{x}}$ is convex (because $F$ has convex values), we have $\left(\lambda \tilde{\rho}_{n}^{1}+(1-\lambda) \tilde{\rho}_{n}^{2}\right) \in \Phi_{n}^{2} x$.

(iii) $\Phi_{n}^{2}(\bar{V})$ is completely continuous.

Let $V$ be a bounded set as in Step 1. For each $x \in \bar{V}$ and $\tilde{\rho}_{n} \in \Phi_{n}^{2} x$, there exists $f \in S_{F, \bar{x}}$ such that

$$
\begin{aligned}
\tilde{\rho}_{n}(t)= & R(t) \varphi(0)+\int_{0}^{t} R(t-s) B u u_{\bar{x}}^{a}(s) d s+\int_{0}^{t} R(t-s) f(s) d w(s) \\
& +\sum_{0<t_{k}<t} R\left(t-t_{k}\right) R\left(\sigma_{n}\right) I_{k}\left(\bar{x}_{t_{k}}\right) .
\end{aligned}
$$

We consider the decomposition $\Phi_{n}^{2}(\overline{\bar{V}})=\Gamma_{n}^{1}(\bar{V})+\Gamma_{n}^{1}(\bar{V})$, where the map $\Gamma_{n}^{1}$ is defined by $\Gamma_{n}^{1} x, x \in \bar{V}$, the set $\tilde{\gamma}_{n}^{1}$ is such that

$$
\tilde{\gamma}_{n}^{1}(t)=R(t) \varphi(0)+\int_{0}^{t} R(t-s) B u_{\bar{x}}^{a}(s) d s+\int_{0}^{t} R(t-s) f(s) d w(s)
$$


and the map $\Gamma_{n}^{2}$ is defined by $\Gamma_{n}^{2} x, x \in \bar{V}$, the set $\tilde{\gamma}_{n}^{2}$ is such that

$$
\tilde{\gamma}_{n}^{2}(t)=\sum_{0<t_{k}<t} R\left(t-t_{k}\right) R\left(\sigma_{n}\right) I_{k}\left(\bar{x}_{t_{k}}\right)
$$

(a) $\Gamma_{n}^{1}(\bar{V})$ is completely continuous.

We begin by showing that $\Gamma_{n}^{1}(\bar{V})$ is equicontinuous. If $x \in \bar{V}$, from Lemma 2.3 it follows that

$$
\left\|\bar{x}_{s}\right\|_{\mathcal{B}}^{p} \leq 2^{p-1}\left(M_{b}\|\varphi\|_{\mathcal{B}}\right)^{p}+2^{p-1} K_{b}^{p} r^{*}:=r^{\prime}
$$

Let $0<\tau_{1}<\tau_{2} \leq b$. For each $x \in \bar{V}$, we have

$$
\begin{aligned}
E\left\|\tilde{\gamma}_{n}^{1}\left(\tau_{2}\right)-\tilde{\gamma}_{n}^{1}\left(\tau_{1}\right)\right\|_{H}^{p} & \\
\leq & 7^{p-1} E\left\|\left[R\left(\tau_{2}\right)-R\left(\tau_{1}\right)\right] \varphi(0)\right\|_{H}^{p} \\
& +7^{p-1} E\left\|\int_{0}^{\tau_{1}-\varepsilon}\left[R\left(\tau_{2}-s\right)-R\left(\tau_{1}-s\right)\right] B u_{\bar{x}}^{a}(s) d s\right\|_{H}^{p} \\
& +7^{p-1} E\left\|\int_{\tau_{1}-\varepsilon}^{\tau_{1}}\left[R\left(\tau_{2}-s\right)-R\left(\tau_{1}-s\right)\right] B u_{\bar{x}}^{a}(s) d s\right\|_{H}^{p} \\
& +7^{p-1} E\left\|\int_{\tau_{1}}^{\tau_{2}} R\left(\tau_{2}-s\right) B u_{\bar{x}}^{a}(s) d s\right\|_{H}^{p} \\
& +7^{p-1} E\left\|\int_{0}^{\tau_{1}-\varepsilon}\left[R\left(\tau_{2}-s\right)-R\left(\tau_{1}-s\right)\right] f(s) d w(s)\right\|_{H}^{p} \\
& +7^{p-1} E\left\|E \int_{\tau_{1}-\varepsilon}^{\tau_{1}}\left[R\left(\tau_{2}-s\right)-R\left(\tau_{1}-s\right)\right] f(s) d w(s)\right\|_{H}^{p} \\
& +7^{p-1} E\left\|\int_{\tau_{1}}^{\tau_{2}} R\left(\tau_{2}-s\right) f(s) d w(s)\right\|_{H}^{p} \\
\leq & 7^{p-1} E\left\|\left[R\left(\tau_{2}\right)-R\left(\tau_{1}\right)\right] \varphi(0)\right\|_{H}^{p} \\
& +7^{p-1} E\left[\int_{0}^{\tau_{1}-\varepsilon}\left\|R\left(\tau_{2}-s\right)-R\left(\tau_{1}-s\right)\right\|_{H}\left\|B u u_{\bar{x}}^{a}(s)\right\|_{H} d s\right]^{p} \\
\leq & 7^{p-1} E\left\|\left[R\left(\tau_{2}\right)-R\left(\tau_{1}\right)\right] \varphi(0)\right\|_{H}^{p} \\
& +7^{p-1} E\left[\int_{\tau_{1}-\varepsilon}^{\tau_{1}}\left\|R\left(\tau_{2}-s\right)-R\left(\tau_{1}-s\right)\right\|_{H}\left\|B u_{\bar{x}}^{a}(s)\right\|_{H} d s\right]^{p} \\
& \left.+7^{p-1} C_{p}\left[\int_{\tau_{1}-\varepsilon}^{\tau_{1}}\left[\left\|R\left(\tau_{2}-s\right)-R\left(\tau_{1}-s\right)\right\|_{H}^{p} E\|f(s)\|_{H}^{p}\right]^{2 / p} d s\right]_{\tau_{1}}^{\tau_{2}}\left\|R\left(\tau_{2}-s\right)\right\|_{H}\left\|B u_{\bar{x}}^{a}(s)\right\|_{H} d s\right]^{p} \\
& +7^{p-1} C_{p}\left[\int_{0}^{\tau_{1}-\varepsilon}\left[\left\|R\left(\tau_{2}-s\right)-R\left(\tau_{1}-s\right)\right\|_{H}^{p} E\|f(s)\|_{H}^{p}\right]^{2 / p} d s\right]^{p / 2} \\
& +\tau_{2}
\end{aligned}
$$




$$
\begin{aligned}
& +7^{p-1} b^{p-1} M_{2}^{p} \int_{0}^{\tau_{1}-\varepsilon}\left\|R\left(\tau_{2}-s\right)-R\left(\tau_{1}-s\right)\right\|_{H}^{p} M_{3} d s \\
& +14^{p-1} M^{p} M_{2}^{p}\left[\int_{\tau_{1}-\varepsilon}^{\tau_{1}} e^{-\delta\left(\tau_{1}-s\right)} d s\right]^{p-1} \int_{\tau_{1}-\varepsilon}^{\tau_{1}} e^{-\delta\left(\tau_{1}-s\right)} M_{3} d s \\
& +7^{p-1} M^{p} M_{2}^{p}\left[\int_{\tau_{1}}^{\tau_{2}} e^{-\delta\left(\tau_{2}-s\right)} d s\right]^{p-1} \int_{\tau_{1}}^{\tau_{2}} e^{-\delta\left(\tau_{2}-s\right)} M_{3} d s \\
& +7^{p-1} C_{p}\left[\int_{0}^{\tau_{1}-\varepsilon}\left[\left\|R\left(\tau_{2}-s\right)-R\left(\tau_{1}-s\right)\right\|_{H}^{p} m_{f}(s) \Theta_{f}\left(\left\|\bar{x}_{s}\right\|_{\mathcal{B}}^{p}\right)\right]^{2 / p} d s\right]^{p / 2} \\
& +7^{p-1} C_{p}\left[\int_{\tau_{1}-\varepsilon}^{\tau_{1}}\left[\left\|R\left(\tau_{2}-s\right)-R\left(\tau_{1}-s\right)\right\|_{H}^{p} m_{f}(s) \Theta_{f}\left(\left\|\bar{x}_{s}\right\|_{\mathcal{B}}^{p}\right)\right]^{2 / p} d s\right]^{p / 2} \\
& +7^{p-1} C_{p}\left[\int_{\tau_{1}}^{\tau_{2}}\left[\left\|R\left(\tau_{2}-s\right)\right\|_{H}^{p} m_{f}(s) \Theta_{f}\left(\left\|\bar{x}_{s}\right\|_{\mathcal{B}}^{p}\right)\right]^{2 / p} d s\right]^{p / 2} \\
& \leq 7^{p-1} E\left\|\left[R\left(\tau_{2}\right)-R\left(\tau_{1}\right)\right] \varphi(0)\right\|_{H}^{p} \\
& +7^{p-1} b^{p-1} M_{2}^{p} \int_{0}^{\tau_{1}-\varepsilon}\left\|R\left(\tau_{2}-s\right)-R\left(\tau_{1}-s\right)\right\|_{H}^{p} M_{3} d s \\
& +14^{p-1} M^{p} M_{2}^{p} \delta^{1-p} \int_{\tau_{1}-\varepsilon}^{\tau_{1}} e^{-\delta\left(\tau_{1}-s\right)} M_{3} d s \\
& +7^{p-1} M^{p} M_{2}^{p} \delta^{1-p} \int_{\tau_{1}}^{\tau_{2}} e^{-\delta\left(\tau_{2}-s\right)} M_{3} d s \\
& +7^{p-1} C_{p} \Theta_{f}\left(r^{\prime}\right)\left[\int_{0}^{\tau_{1}-\varepsilon}\left[\left\|R\left(\tau_{2}-s\right)-R\left(\tau_{1}-s\right)\right\|_{H}^{p} m_{f}(s)\right]^{2 / p} d s\right]^{p / 2} \\
& +14^{p-1} C_{p} M^{p} \Theta_{f}\left(r^{\prime}\right)\left[\int_{\tau_{1}-\varepsilon}^{\tau_{1}} e^{-\left[\frac{2(p-1)}{p-2}\right] \delta\left(\tau_{1}-s\right)} d s\right]^{p / 2-1} \int_{\tau_{1}-\varepsilon}^{\tau_{1}} e^{-\delta\left(\tau_{1}-s\right)} m_{f}(s) d s \\
& +7^{p-1} C_{p} M^{p} \Theta_{f}\left(r^{\prime}\right)\left[\int_{\tau_{1}}^{\tau_{2}} e^{-\left[\frac{2(p-1)}{p-2}\right] \delta\left(\tau_{2}-s\right)} d s\right]^{p / 2-1} \int_{\tau_{1}}^{\tau_{2}} e^{-\delta\left(\tau_{2}-s\right)} m_{f}(s) d s \\
& \leq 7^{p-1} E\left\|\left[R\left(\tau_{2}\right)-R\left(\tau_{1}\right)\right] \varphi(0)\right\|_{H}^{p} \\
& +7^{p-1} b^{p-1} M_{2}^{p} \int_{0}^{\tau_{1}-\varepsilon}\left\|R\left(\tau_{2}-s\right)-R\left(\tau_{1}-s\right)\right\|_{H}^{p} M_{3} d s \\
& +14^{p-1} M^{p} M_{2}^{p} \delta^{1-p} \int_{\tau_{1}-\varepsilon}^{\tau_{1}} e^{-\delta\left(\tau_{1}-s\right)} M_{3} d s+7^{p-1} M^{p} M_{2}^{p} \delta^{1-p} \int_{\tau_{1}}^{\tau_{2}} e^{-\delta\left(\tau_{2}-s\right)} M_{3} d s \\
& +7^{p-1} C_{p} \Theta_{f}\left(r^{\prime}\right)\left[\int_{0}^{\tau_{1}-\varepsilon}\left[\left\|R\left(\tau_{2}-s\right)-R\left(\tau_{1}-s\right)\right\|_{H}^{p} m_{f}(s)\right]^{2 / p} d s\right]^{p / 2} \\
& +14^{p-1} C_{p} M^{p} \Theta_{f}\left(r^{\prime}\right)\left[\frac{2 \delta(p-1)}{p-2}\right]^{1-p / 2} \int_{\tau_{1}-\varepsilon}^{\tau_{1}} e^{-\delta\left(\tau_{1}-s\right)} m_{f}(s) d s \\
& +7^{p-1} C_{p} M^{p} \Theta_{f}\left(r^{\prime}\right)\left[\frac{2 \delta(p-1)}{p-2}\right]^{1-p / 2} \int_{\tau_{1}}^{\tau_{2}} e^{-\delta\left(\tau_{2}-s\right)} m_{f}(s) d s
\end{aligned}
$$

where

$$
\begin{aligned}
E\left\|u_{\bar{x}}^{a}(s)\right\|_{H}^{p} \leq & 5^{p-1} M_{*}^{p} \frac{1}{a^{p}} M_{2}^{p} b^{p} N_{*}^{p}\left\{4 ^ { p - 1 } \left[E\left\|x_{0}\right\|_{H}^{p}+C_{p} b^{p / 2-1} \int_{0}^{b} E\|\tilde{\phi}(\eta)\|_{H}^{p} d \eta\right.\right. \\
& \left.+2^{p-1} M^{p} e^{-p \delta b}\left[\left(\tilde{H}\|\varphi\|_{\mathcal{B}}\right)^{p}+\left\|(-A)^{-\beta}\right\|_{H}^{p} M^{p} e^{-p \delta \sigma_{n}} L_{G}\left(\|\varphi\|_{\mathcal{B}}^{p}+1\right)\right]\right]
\end{aligned}
$$




$$
\begin{aligned}
& +\left(4^{p-1}\left\|(-A)^{-\beta}\right\|_{H}^{p}+M_{1-\beta}^{p} \frac{b^{p \beta}}{1-p(1-\beta)}+M_{1-\beta}^{p} M_{1}^{p} \frac{b^{p(\beta+1)}}{1-p(1-\beta)}\right) \\
& \times M^{p} e^{-p \delta \sigma_{n}} L_{G}\left(r^{\prime}+1\right)+M_{*}^{p} \Theta_{f}\left(r^{\prime}\right) \int_{0}^{b} e^{p \delta \eta} m_{f}(\eta) d \eta \\
& \left.+m^{p-1} M_{*}^{p} M^{p} e^{-p \delta \sigma_{n}} N_{*}^{p} \sum_{k=1}^{m}\left[C_{1}+\left(c_{k}+\epsilon_{k}\right) r^{\prime}\right]\right\}:=M_{3}, \\
& M_{*}=M \max \left\{1, e^{-\delta b}\right\} .
\end{aligned}
$$

From the above inequalities, we see that the right-hand side of $E\left\|\tilde{\gamma}_{n}^{1}\left(\tau_{2}\right)-\tilde{\gamma}_{n}^{1}\left(\tau_{1}\right)\right\|_{H}^{p}$ tends to zero independent of $x \in \bar{V}$ as $\tau_{2}-\tau_{1} \rightarrow 0$ with $\varepsilon$ being sufficiently small since $R(t)$ is a continuous operator, and the compactness for $t>0$ implies the continuity in the uniform operator topology. Thus the set $\left\{\Gamma_{n}^{1} x: x \in \bar{V}\right\}$ is equicontinuous. The equicontinuities for the cases $\tau_{1}<\tau_{2} \leq 0$ or $\tau_{1} \leq 0 \leq \tau_{2} \leq b$ are very simple.

Now we prove that $\Gamma_{n}^{1}(\bar{V})(t)=\left\{\tilde{\gamma}_{n}^{1}(t): \tilde{\gamma}_{n}^{1}(t) \in \Gamma_{n}^{1}(\bar{V})\right\}$ is relatively compact for every $t \in$ $[0, b]$. Let $0<t \leq s \leq b$ be fixed, and let $\varepsilon$ be a real number satisfying $0<\varepsilon<t$. For $x \in \bar{V}$, we define

$$
\tilde{\gamma}_{n, \varepsilon}^{1}(t)=R(t) \varphi(0)+\int_{0}^{t-\varepsilon} R(t-s) B u_{\bar{x}}^{a}(s) d s+\int_{0}^{t-\varepsilon} R(t-s) f(s) d w(s)
$$

where $f \in S_{F, \bar{x}}$. Using the compactness of $R(t)$ for $t>0$, we deduce that the set $U_{\varepsilon}(t)=$ $\left\{\tilde{\gamma}_{n, \varepsilon}^{1}(t): x \in \bar{V}\right\}$ is relatively compact in $H$ for every $\varepsilon, 0<\varepsilon<t$. Moreover, for every $x \in \bar{V}$, we have

$$
\begin{aligned}
& E\left\|\tilde{\gamma}_{n}^{1}(t)-\tilde{\gamma}_{n, \varepsilon}^{1}(t)\right\|_{H}^{p} \\
& \leq 2^{p-1} E\left\|\int_{t-\varepsilon}^{t} R(t-s) B u_{\bar{x}}^{a}(s) d s\right\|_{H}^{p} \\
& \quad+2^{p-1} E\left\|\int_{t-\varepsilon}^{t} R(t-s) f(s) d w(s)\right\|_{H}^{p} \\
& \leq 2^{p-1} M^{p} M_{2}^{p} \delta^{1-p} \int_{t-\varepsilon}^{t} e^{-\delta(t-s)} M_{3} d s \\
& \quad+2^{p-1} C_{p} M^{p} \Theta_{f}\left(r^{\prime}\right)\left[\frac{2 \delta(p-1)}{p-2}\right]^{1-p / 2} \int_{t-\varepsilon}^{t} e^{-\delta(t-s)} m_{f}(s) d s .
\end{aligned}
$$

The right-hand side of the above inequality tends to zero as $\varepsilon \rightarrow 0$. Since there are relatively compact sets arbitrarily close to the set $U(t)=\left\{\tilde{\gamma}_{n}^{1}(t): x \in \bar{V}\right\}$, hence the set $U(t)$ is relatively compact in $H$. By the Arzelá-Ascoli theorem, we conclude that $\Gamma_{n}^{1}(\bar{V})$ is completely continuous.

(b) $\Gamma_{n}^{2}$ is completely continuous.

We begin by showing that $\Gamma_{n}^{2}(\bar{V})$ is equicontinuous. For each $x \in \bar{V}, t \in(0, b)$ is fixed, $t \in\left[t_{i}, t_{i+1}\right]$ and $\tilde{\gamma}_{n}^{2} \in \Gamma_{n}^{2} x$ such that

$$
\tilde{\gamma}_{n}^{2}(t)=\sum_{0<t_{k}<t} R\left(t-t_{k}\right) R\left(\sigma_{n}\right) I_{k}\left(\bar{x}_{t_{k}}\right)
$$


Next, for $\tau_{1} \leq s<t \leq \tau_{2}, \varepsilon>0$, we have, using the property of a compact operator,

$$
\begin{aligned}
E\left\|\left[\widehat{\widetilde{\gamma}}_{n}^{2}\right]_{i}\left(\tau_{2}\right)-\left[\widehat{\widetilde{\gamma}}_{n}^{2}\right]_{i}\left(\tau_{1}\right)\right\|_{H}^{p} & =E\left\|\sum_{0<t_{k}<t}\left[R\left(\tau_{2}-t_{k}\right)-R\left(\tau_{1}-t_{k}\right)\right] R\left(\sigma_{n}\right) I_{k}\left(\bar{x}_{t_{k}}\right)\right\|_{H}^{p} \\
& \leq m^{p-1} \sum_{k=1}^{m} E\left\|\left[R\left(\tau_{2}-t_{k}\right)-R\left(\tau_{1}-t_{k}\right)\right] R\left(\sigma_{n}\right) I_{k}\left(\bar{x}_{t_{k}}\right)\right\|_{H}^{p}
\end{aligned}
$$

As $\tau_{2}-\tau_{1} \rightarrow 0$, the right-hand side of the above inequality tends to zero independently of $x$ due to the set $\left\{R\left(\sigma_{n}\right) I_{k}\left(\bar{x}_{t_{k}}\right): x \in \bar{V}\right\}$ being relatively compact in $H$ and the strong continuity of $R(\cdot)$. So $\left[\widehat{\widetilde{\gamma}}_{n}^{2}\right]_{i}, i=1,2, \ldots, m$, are equicontinuous.

Now we prove that $\left[\widehat{\tilde{\gamma}}_{n}^{2}\right]_{i}(t), i=1,2, \ldots, m$, is relatively compact for every $t \in[0, b]$.

From the following relations

$$
\left[\widehat{\widetilde{\gamma}}_{n}^{2}\right]_{i}(t)=\sum_{0<t_{k}<t} R\left(t-t_{k}\right) R\left(\sigma_{n}\right) I_{k}\left(\bar{x}_{t_{k}}\right) \in \sum_{k=1}^{m} R\left(t-t_{k}\right) R\left(\sigma_{n}\right) I_{k}(\bar{V}(0, H))
$$

we conclude that $\left[\widehat{\tilde{\gamma}_{n}^{2}(\bar{V})}\right]_{i}(t), i=1,2, \ldots, m$, is relatively compact for every $t \in\left[t_{i}, t_{i+1}\right]$. By Lemma 2.6, we infer that $\Gamma_{n}^{2}(\bar{V})$ is relatively compact. Now an application of the ArzeláAscoli theorem justifies the relative compactness of $\Gamma_{n}^{2}(\bar{V})$. Therefore, $\Gamma_{n}^{2}(\bar{V})$ is completely continuous, and hence $\Phi_{n}^{2}(\bar{V})$ is completely continuous.

As a consequence of the above Steps 1-3, we conclude that $\Phi_{n}=\Phi_{n}^{1}+\Phi_{n}^{2}$ is a condensing map. All of the conditions of Lemma 2.8 are satisfied, we deduce that $\Phi_{n}$ has a fixed point $x_{n} \in \mathcal{B P C}$, which is in turn a mild solution of problem (3.2)-(3.4). Then we have

$$
\begin{aligned}
x_{n}(t)= & R(t)\left[\varphi(0)-R\left(\sigma_{n}\right) G(0, \varphi)\right]+R\left(\sigma_{n}\right) G\left(t, \bar{x}_{n, t}\right) \\
& +\int_{0}^{t} A R(t-s) R\left(\sigma_{n}\right) G\left(s, \bar{x}_{n, s}\right) d s \\
& +\int_{0}^{t} A R(t-s) \int_{0}^{s} h(s-\tau) R\left(\sigma_{n}\right) G\left(\tau, \bar{x}_{n, \tau}\right) d \tau d s \\
& +\int_{0}^{t} R(t-s) B u_{\bar{x}_{n}}^{a}(s) d s+\int_{0}^{t} R(t-s) f_{n}(s) d w(s) \\
& +\sum_{0<t_{k}<t} R\left(t-t_{k}\right) R\left(\sigma_{n}\right) I_{k}\left(\bar{x}_{n, t_{k}}\right), \quad t \in J
\end{aligned}
$$

for $t \in[0, b]$ and some $f_{n} \in S_{F, \bar{x}_{n}}$.

Next we will show that the set $\left\{x_{n}: n \in \mathbb{N}\right\}$ is relatively compact in $\mathcal{B P C}$. We consider the decomposition $x_{n}=x_{n}^{1}+x_{n}^{2}$, where

$$
\begin{aligned}
x_{n}^{1}(t)= & R(t)\left[\varphi(0)-R\left(\sigma_{n}\right) G(0, \varphi)\right]+R\left(\sigma_{n}\right) G\left(t, \bar{x}_{n, t}\right) \\
& +\int_{0}^{t} A R(t-s) R\left(\sigma_{n}\right) G\left(s, \bar{x}_{n, s}\right) d s \\
& +\int_{0}^{t} A R(t-s) \int_{0}^{s} h(s-\tau) R\left(\sigma_{n}\right) G\left(\tau, \bar{x}_{n, \tau}\right) d \tau d s \\
& +\int_{0}^{t} R(t-s) B u_{\bar{x}_{n}}^{a}(s) d s+\int_{0}^{t} R(t-s) f_{n}(s) d w(s)
\end{aligned}
$$


for some $f_{n} \in S_{F, \bar{x}_{n}}$, and

$$
x_{n}^{2}(t)=\sum_{0<t_{k}<t} R\left(t-t_{k}\right) R\left(\sigma_{n}\right) I_{k}\left(\bar{x}_{n, t_{k}}\right) .
$$

Step $4 .\left\{x_{n}^{1}(t): n \in \mathbb{N}\right\}$ is relatively compact in $\mathcal{B P C}$.

(i) $\left\{x_{n}^{1}: n \in \mathbb{N}\right\}$ is equicontinuous on $J$.

For $\varepsilon>0, x_{n} \in \bar{V}$, there exists a constant $0<\tilde{\eta}<\varepsilon$ such that for all $t \in(0, b]$ and $\xi \in(0, \tilde{\eta})$ with $t+\xi \leq b$, we have

$$
\begin{aligned}
& E\left\|x_{n}^{1}(t+\xi)-x_{n}^{1}(t)\right\|_{H}^{p} \\
& \leq 10^{p-1} E\left\|[R(t+\xi)-R(t)]\left[\varphi(0)-R\left(\sigma_{n}\right) G(0, \varphi)\right]\right\|_{H}^{p} \\
& +10^{p-1} E\left\|R\left(\sigma_{n}\right)\left[G\left(t+\xi, \bar{x}_{n, t+\xi},\right)-G\left(t, \bar{x}_{n, t}\right)\right]\right\|_{H}^{p} \\
& +10^{p-1} E\left\|\int_{t}^{t+\xi} A R(t+\xi-s) R\left(\sigma_{n}\right) G\left(s, \bar{x}_{n, s}\right) d s\right\|_{H}^{p} \\
& +10^{p-1} E\left\|\int_{0}^{t} A[R(t+\xi-s)-R(t-s)] R\left(\sigma_{n}\right) G\left(s, \bar{x}_{n, s}\right) d s\right\|_{H}^{p} \\
& +10^{p-1} E\left\|\int_{t}^{t+\xi} A R(t+\xi-s) \int_{0}^{s} h(s-\tau) R\left(\sigma_{n}\right) G\left(\tau, \bar{x}_{n, \tau}\right) d \tau d s\right\|_{H}^{p} \\
& +10^{p-1} E\left\|\int_{0}^{t} A[R(t+\xi-s)-R(t-s)] \int_{0}^{s} h(s-\tau) R\left(\sigma_{n}\right) G\left(\tau, \bar{x}_{n, \tau}\right) d \tau d s\right\|_{H}^{p} \\
& +10^{p-1} E\left\|\int_{t}^{t+\xi} R(t+\xi-s) B u_{\bar{x}_{n}}^{a}(s) d s\right\|_{H}^{p} \\
& +10^{p-1} E\left\|\int_{0}^{t}[R(t+\xi-s)-R(t-s)] B u_{\bar{x}_{n}}^{a}(s) d s\right\|_{H}^{p} \\
& +10^{p-1} E\left\|\int_{t}^{t+\xi} R(t+\xi-s) f_{n}(s) d w(s)\right\|_{H}^{p} \\
& +10^{p-1} E\left\|\int_{0}^{t}[R(t+\xi-s)-R(t-s)] f_{n}(s) d w(s)\right\|_{H}^{p} \\
& \leq 10^{p-1} E\left\|[R(t+\xi)-R(t)] R\left(\sigma_{n}\right) G(0, \varphi)\right\|_{H}^{p} \\
& +10^{p-1}\left\|(-A)^{-\beta}\right\|_{H}^{p} M^{p} e^{-p \delta \sigma_{n}} L_{G}\left[\xi+\left\|\bar{x}_{n, t+\xi}-\bar{x}_{n, t}\right\|_{\mathcal{B}}^{p}\right] \\
& +10^{p-1} M_{1-\beta}^{p} \xi^{p-1} \int_{t}^{t+\xi}(t+\xi-s)^{-p(1-\beta)} M^{p} e^{-p \delta \sigma_{n}} L_{G}\left(r^{\prime}+1\right) d s \\
& +10^{p-1} b^{p-1} \int_{0}^{t}\left\|(-A)^{-\beta}[R(t+\xi-s)-R(t-s)]\right\|_{H}^{p} M^{p} e^{-p \delta \sigma_{n}} L_{G}\left(r^{\prime}+1\right) d s \\
& +10^{p-1} M_{1-\beta}^{p} M_{1}^{p} b^{p-1} \int_{t}^{t+\xi}(t+\xi-s)^{-p(1-\beta)} M^{p} e^{-p \delta \sigma_{n}} L_{G}\left(r^{\prime}+1\right) d s \\
& +10^{p-1} b^{2 p-1} M_{1}^{p} \int_{0}^{t}\left\|(-A)^{1-\beta}[R(t+\xi-s)-R(t-s)]\right\| M^{p} e^{-p \delta \sigma_{n}} L_{G}\left(r^{\prime}+1\right) d s \\
& +10^{p-1} M^{p} M_{2}^{p} \delta^{1-p} \int_{t}^{t+\xi} e^{-\delta(t-s)} M_{3} d s
\end{aligned}
$$




$$
\begin{aligned}
& +10^{p-1} M_{2}^{p} b^{p-1} \int_{0}^{t}\|R(t+\xi-s)-R(t-s)\|_{H}^{p} M_{3} d s \\
& +10^{p-1} C_{p} M^{p} \Theta_{f}\left(r^{\prime}\right)\left[\frac{2 \delta(p-1)}{p-2}\right]^{1-p / 2} \int_{t}^{t+\xi} e^{-\delta(t-s)} m_{f}(s) d s \\
& +10^{p-1} C_{p} \Theta_{f}\left(r^{\prime}\right)\left[\int_{0}^{t}\left[\|R(t+\xi-s)-R(t-s)\|_{H}^{p} m_{f}(s)\right]^{2 / p} d s\right]^{p / 2} .
\end{aligned}
$$

Using the compact operator property, we can choose $\xi \in(0, t)$ such that

$$
10^{p-1} E\left\|[R(t+\xi)-R(t)] R\left(\sigma_{n}\right) G(0, \varphi)\right\|_{H}^{p}<\frac{\varepsilon}{10}
$$

and

$$
\begin{aligned}
& 10^{p-1}\left\|(-A)^{-\beta}\right\|_{H}^{p} M^{p} e^{-p \delta \sigma_{n}} L_{G}\left[\xi+\left\|\bar{x}_{n, t+\xi}-\bar{x}_{n, t}\right\|_{\mathcal{B}}^{p}\right]<\frac{\varepsilon}{10}, \\
& 10^{p-1} M_{1-\beta}^{p} \xi^{p-1} \int_{t}^{t+\xi}(t+\xi-s)^{-p(1-\beta)} M^{p} e^{-p \delta \sigma_{n}} L_{G}\left(r^{\prime}+1\right) d s<\frac{\varepsilon}{10}, \\
& 10^{p-1} b^{p-1} \int_{0}^{t}\left\|(-A)^{-\beta}[R(t+\xi-s)-R(t-s)]\right\|_{H}^{p} M^{p} e^{-p \delta \sigma_{n}} L_{G}\left(r^{\prime}+1\right) d s<\frac{\varepsilon}{10}, \\
& 10^{p-1} M_{1-\beta}^{p} M_{1}^{p} b^{p-1} \int_{t}^{t+\xi}(t+\xi-s)^{-p(1-\beta)} M^{p} e^{-p \delta \sigma_{n}} L_{G}\left(r^{\prime}+1\right) d s<\frac{\varepsilon}{10}, \\
& 10^{p-1} b^{2 p-1} M_{1}^{p} \int_{0}^{t}\left\|(-A)^{1-\beta}[R(t+\xi-s)-R(t-s)]\right\| M^{p} e^{-p \delta \sigma_{n}} L_{G}\left(r^{\prime}+1\right) d s<\frac{\varepsilon}{10}, \\
& 10^{p-1} M^{p} M_{2}^{p} \delta^{1-p} \int_{t}^{t+\xi} e^{-\delta(t-s)} M_{3} d s<\frac{\varepsilon}{10}, \\
& 10^{p-1} M_{2}^{p} b^{p-1} \int_{0}^{t}\|R(t+\xi-s)-R(t-s)\|_{H}^{p} M_{3} d s<\frac{\varepsilon}{10}, \\
& 10^{p-1} C_{p} M^{p} \Theta_{f}\left(r^{\prime}\right)\left[\frac{2 \delta(p-1)}{p-2}\right]^{1-p / 2} \int_{t}^{t+\xi} e^{-\delta(t-s)} m_{f}(s) d s<\frac{\varepsilon}{10}, \\
& 10^{p-1} C_{p} \Theta_{f}\left(r^{\prime}\right)\left[\int_{0}^{t}\left[\|R(t+\xi-s)-R(t-s)\|_{H}^{p} m_{f}(s)\right]^{2 / p} d s\right]^{p / 2}<\frac{\varepsilon}{10} .
\end{aligned}
$$

By (3.11)-(3.20) one has

$$
E\left\|x_{n}^{1}(t+\xi)-x_{n}^{1}(t)\right\|_{H}^{p}<\varepsilon
$$

Therefore, $\left\{x_{n}^{1}(t): n \in \mathbb{N}\right\}$ is equicontinuous for $t \in(0, b]$. Clearly, $\left\{x_{n}^{1}(0): n \in \mathbb{N}\right\}$ is equicontinuous.

(ii) $\left\{x_{n}^{1}(t): n \in \mathbb{N}\right\}$ is relatively compact in $H$.

Let $t \in(0, b], \varepsilon>0, x_{n} \in \bar{V}$, there exists $\xi \in(0, t)$ such that

$$
\begin{aligned}
& E\left\|x_{n}^{1}(t)-x_{n}^{\xi}(t)\right\|_{H}^{p} \\
& \leq 4^{p-1} E\left\|\int_{t-\xi}^{t} A R(t-s) R\left(\sigma_{n}\right) G\left(s, \bar{x}_{n, s}\right) d s\right\|_{H}^{p} \\
& \quad+4^{p-1} E\left\|\int_{t-\xi}^{t} A R(t-s) \int_{0}^{s} h(s-\tau) R\left(\sigma_{n}\right) G\left(\tau, \bar{x}_{n, \tau}\right) d \tau d s\right\|_{H}^{p}
\end{aligned}
$$




$$
\begin{aligned}
& +4^{p-1} E\left\|\int_{t-\xi}^{t} R(t-s) B u_{\bar{x}_{n}}^{a}(s) d s\right\|_{H}^{p}+4^{p-1} E\left\|\int_{t-\xi}^{t} R(t-s) f_{n}(s) d w(s)\right\|_{H}^{p} \\
\leq & 4^{p-1} \xi^{p-1} M_{1-\beta}^{p} M^{p} e^{-p \delta \sigma_{n}} L_{G}\left(r^{\prime}+1\right) \int_{t-\xi}^{t}(t-s)^{-p(1-\beta)} d s \\
& +4^{p-1} \xi^{p-1} M_{1-\beta}^{p} M_{1}^{p} b^{p} M^{p} e^{-p \delta \sigma_{n}} L_{G}\left(r^{\prime}+1\right) \int_{t-\xi}^{t}(t-s)^{-p(1-\beta)} d s \\
& +4^{p-1} M^{p} M_{2}^{p} \delta^{1-p} \int_{t-\xi}^{t} e^{-\delta(t-s)} M_{3} d s \\
& +4^{p-1} C_{p} M^{p} \Theta_{f}\left(r^{\prime}\right)\left[\frac{2 \delta(p-1)}{p-2}\right]^{1-p / 2} \int_{t}^{t-\xi} e^{-\delta(t-s)} m_{f}(s) d s<\varepsilon
\end{aligned}
$$

where

$$
\begin{aligned}
x_{n}^{\xi}(t)= & R(t)\left[\varphi(0)-R\left(\sigma_{n}\right) G(0, \varphi)\right]+R\left(\sigma_{n}\right) G\left(t, \bar{x}_{n, t}\right) \\
& +\int_{0}^{t-\xi} A R(t-s) R\left(\sigma_{n}\right) G\left(s, \bar{x}_{n, s}\right) d s \\
& +\int_{0}^{t-\xi} A R(t-s) \int_{0}^{s} h(s-\tau) R\left(\sigma_{n}\right) G\left(\tau, \bar{x}_{n, \tau}\right) d \tau d s \\
& +\int_{0}^{t-\xi} S(t-s) B u_{\bar{x}_{n}}^{a}(s) d s+\int_{0}^{t-\xi} S(t-s) f_{n}(s) d w(s)
\end{aligned}
$$

for some $f_{n} \in S_{F, \bar{x}_{n}}$. From (H4) we obtain that $G\left(t, \bar{x}_{n, t}\right)$ is bounded in $H$. By the compactness of $R(t)$ for $t>0$, we see that the set $\left\{x_{n}^{\xi}(t): n \in \mathbb{N}\right\}$ is relatively compact in $H$. Combining the above inequality, one has that $\left\{x_{n}^{1}(t): n \in \mathbb{N}\right\}$ is relatively compact in $H$.

Step 5 . $\left\{x_{n}^{2}(t): n \in \mathbb{N}\right\}$ is relatively compact in $\mathcal{B P C}$.

(i) $\left\{x_{n}^{2}: n \in \mathbb{N}\right\}$ is equicontinuous on $J$.

For any $\varepsilon>0$ and $0<t<b$, since $R\left(\sigma_{n}\right)$ is a compact operator, we find that the sets $W=$ $\left\{R\left(\sigma_{n}\right) I_{k}\left(\bar{x}_{n, t_{k}}\right): x_{n} \in \bar{V}\right\}$ are relatively compact in $H$. From the strong continuity of $(R(t))_{t \geq 0}$, for $\varepsilon>0$, we can choose $0<\tilde{\eta}<b-t$ such that

$$
E\|(R(t+\xi)-R(t)) \tilde{v}\|_{H}^{p}<\frac{\varepsilon}{m^{p}}, \quad \tilde{v} \in W
$$

when $|\xi|<\tilde{\eta}$. For each $x_{n} \in \bar{V}, t \in(0, b)$ is fixed, $t \in\left[t_{i}, t_{i+1}\right]$, such that

$$
\begin{aligned}
& E\left\|\left[\widehat{x_{n}^{2}}\right]_{i}(t+\xi)-\left[\widehat{x_{n}^{2}}\right]_{i}(t)\right\|_{H}^{p} \\
& \quad=E\left\|\sum_{0<t_{k}<t}\left[R\left(t+\xi-t_{k}\right)-R\left(t-t_{k}\right)\right] R\left(\sigma_{n}\right) I_{k}\left(\bar{x}_{n, t_{k}}\right)\right\|_{H}^{p} \\
& \quad \leq m^{p-1} \sum_{k=1}^{m} E\left\|\left[R\left(t+\xi-t_{k}\right)-R\left(t-t_{k}\right)\right] R\left(\sigma_{n}\right) I_{k}\left(\bar{x}_{n, t_{k}}\right)\right\|_{H}^{p}<\varepsilon .
\end{aligned}
$$

As $\xi \rightarrow 0$ and $\varepsilon$ sufficiently small, the right-hand side of the above inequality tends to zero independently of $x_{n}$, so $\left[\widehat{x}_{n}^{2}\right]_{i}, i=1,2, \ldots, m$, are equicontinuous.

(ii) $\left\{x_{n}^{2}(t): n \in \mathbb{N}\right\}$ is relatively compact in $H$. 
For $t \in\left[t_{k}, t_{k+1}\right], k=1, \ldots, m$, and $x_{n} \in \bar{V}$, we have that there exists $r^{\prime \prime}>0$ such that

$$
\left[\widehat{x_{n}^{2}}\right]_{k}(t) \in \begin{cases}\sum_{i=1}^{k} R\left(t-t_{i}\right) R\left(\sigma_{n}\right) I_{i}\left(\bar{x}_{n, t_{i}}\right), & t \in\left(t_{k}, t_{k+1}\right), x_{n} \in B_{r^{\prime \prime}}(0, \mathcal{B P C}) \\ \sum_{i=1}^{k} R\left(t_{k+1}-t_{i}\right) R\left(\sigma_{n}\right) I_{i}\left(\bar{x}_{n, t_{i}}\right), & t=t_{k+1}, x_{n} \in B_{r^{\prime \prime}}(0, \mathcal{B P C}) \\ \sum_{i=1}^{k-1} R\left(t_{k}-t_{i}\right) R\left(\sigma_{n}\right) I_{i}\left(\bar{x}_{n, t_{i}}\right) & \\ +R\left(\sigma_{n}\right) I_{k}\left(\bar{x}_{n, t_{i}}\right), & t=t_{k}, x_{n} \in B_{r^{\prime \prime}}(0, \mathcal{B P C})\end{cases}
$$

where $B_{r^{\prime \prime}}(0, \mathcal{B P C})$ is a closed ball of radius $r^{\prime \prime}$. One has that $\left[\widehat{x_{n}^{2}}\right]_{k}(t), k=1,2, \ldots, m$, is relatively compact for every $t \in\left[t_{k}, t_{k+1}\right]$, and $\left\{x_{n}^{2}(t): n \in \mathbb{N}\right\}$ is relatively compact in $H$.

These facts imply the relative compactness of $\left\{x_{n}: n \in \mathbb{N}\right\}$ in $\mathcal{B P C}$. Therefore, without loss of generality, we may suppose that

$$
x_{n} \rightarrow x_{*} \in \mathcal{B P C} \quad \text { as } n \rightarrow \infty
$$

Obviously, $x_{*} \in \mathcal{B P C}$, taking limits in (3.8) one has

$$
\begin{aligned}
x_{*}(t)= & R(t)[\varphi(0)-G(0, \varphi)]+G\left(t, \bar{x}_{*, t}\right)+\int_{0}^{t} A R(t-s) G\left(s, \bar{x}_{*, s}\right) d s \\
& +\int_{0}^{t} A R(t-s) \int_{0}^{s} h(s-\tau) G\left(\tau, \bar{x}_{*, \tau}\right) d \tau d s+\int_{0}^{t} R(t-s) B u_{\bar{x}_{*}}^{a}(s) d s \\
& +\int_{0}^{t} R(t-s) f_{*}(s) d w(s)+\sum_{0<t_{k}<t} R\left(t-t_{k}\right) I_{k}\left(\bar{x}_{*, t_{k}}\right), \quad t \in J
\end{aligned}
$$

for $t \in[0, b]$, and some $f_{*} \in S_{F, \bar{x}_{*}}$, which implies that $x_{*}$ is a mild solution of problem (1.1)(1.3), and the proof of Theorem 3.1 is complete.

\section{Approximate controllability of impulsive stochastic control systems}

In this section, we present our main result on the approximate controllability of system (1.1)-(1.3). To do this, we also need the following assumptions.

(B1) There exists a constant $\beta$ with $0<\beta<1$ such that $G: J \times \mathcal{B} \rightarrow H_{\beta}$ is a continuous function, and $(-A)^{\beta} G: J \times \mathcal{B} \rightarrow H$. Moreover, there exists a constant $\widetilde{C}_{1}>0$ such that

$$
E\left\|(-A)^{\beta} G(t, \psi)\right\|_{H}^{p} \leq \widetilde{C}_{1}
$$

for $0 \leq t \leq b, \psi \in \mathcal{B}$.

(B2) There exists a constant $\widetilde{C}_{2}>0$ such that

$$
\|F(t, \psi)\|_{H}^{p} \leq \widetilde{C}_{2}
$$

for $0 \leq t \leq b, \psi \in \mathcal{B}$, where

$$
\|F(t, \psi)\|_{H}^{p}=\sup \left\{E\|f\|_{H}^{p}: f \in F(t, \psi)\right\}
$$

Theorem 4.1 Assume that the assumptions of Theorem 3.1 hold and, in addition, hypotheses (S1), (B1) and (B2) are satisfied. Then system (1.1)-(1.3) is approximately controllable on $J$. 
Proof Let $x^{a}(\cdot)$ be a fixed point of $\Phi$ in $\mathcal{B P C}$. By Theorem 3.1, any fixed point of $\Phi$ is a mild solution of system (1.1)-(1.3). This means that there is $x^{a} \in \Phi\left(x^{a}\right)$, that is, there is $f \in S_{F, \bar{x}^{a}}$ such that

$$
\begin{aligned}
x^{a}(t)= & R(t)[\varphi(0)-G(0, \varphi)]+G\left(t, \bar{x}_{t}^{a}\right)+\int_{0}^{t} A R(t-s) G\left(s, \bar{x}_{s}^{a}\right) d s \\
& +\int_{0}^{t} A R(t-s) \int_{0}^{s} h(s-\tau) G\left(\tau, \bar{x}_{\tau}^{a}\right) d \tau d s \\
& +\int_{0}^{t} R(t-s) B u_{\bar{x}^{a}}^{a}(s) d s+\int_{0}^{t} R(t-s) f(s) d w(s) \\
& +\sum_{0<t_{k}<t} R\left(t-t_{k}\right) I_{k}\left(\bar{x}_{t_{k}}^{a}\right), \quad t \in J
\end{aligned}
$$

where

$$
\begin{aligned}
u_{\bar{x}}^{a}(s)= & B^{*} R^{*}(b-s)\left(a I+\Gamma_{0}^{b}\right)^{-1}\left[E \tilde{x}_{b}+\int_{0}^{b} \tilde{\phi}(\eta) d w(\eta)-R(b)[\varphi(0)-G(0, \varphi)]\right. \\
& \left.-G\left(b, \bar{x}_{b}^{a}\right)\right]-B^{*} R^{*}(b-s) \int_{0}^{b}\left(a I+\Gamma_{\eta}^{b}\right)^{-1} A R(b-\eta) G\left(\eta, \bar{x}_{\eta}^{a}\right) d \eta \\
& -B^{*} R^{*}(b-s) \int_{0}^{b}\left(a I+\Gamma_{\eta}^{b}\right)^{-1} A R(b-\eta) \int_{0}^{\eta} h(\eta-v) G\left(v, \bar{x}_{v}^{a}\right) d v d \eta \\
& -B^{*} R^{*}(b-s) \int_{0}^{b}\left(a I+\Gamma_{\eta}^{b}\right)^{-1} R(b-\eta) f(\eta) d w(\eta) \\
& -B^{*} R^{*}(b-s)\left(a I+\Gamma_{\eta}^{b}\right)^{-1} \sum_{k=1}^{m} R\left(b-t_{k}\right) I_{k}\left(\bar{x}_{t_{k}}^{a}\right), \quad t \in J
\end{aligned}
$$

and by using the stochastic Fubini theorem, it is easy to see that

$$
\begin{aligned}
x^{a}(b)= & R(t)[\varphi(0)-G(0, \varphi)]+G\left(b, \bar{x}_{b}^{a}\right)+\int_{0}^{b} A R(t-s) G\left(s, \bar{x}_{s}^{a}\right) d s \\
& +\int_{0}^{b} A R(t-s) \int_{0}^{s} h(s-\tau) G\left(\tau, \bar{x}_{\tau}^{a}\right) d \tau d s \\
& +\int_{0}^{b} R(b-s) B u_{\bar{x}^{a}}^{a}(s) d s+\int_{0}^{b} R(t-s) f(s) d w(s)+\sum_{0<t_{k}<b} R\left(b-t_{k}\right) I_{k}\left(\bar{x}_{t_{k}}^{a}\right) \\
= & x_{b}-a\left(a I+\Gamma_{0}^{b}\right)^{-1}\left[E \tilde{x}_{b}+\int_{0}^{b} \tilde{\phi}(\eta) d w(\eta)-R(b)[\varphi(0)-G(0, \varphi)]\right. \\
& \left.-G\left(b, x_{b}^{a}\right)\right]-a \int_{0}^{b} A\left(a I+\Gamma_{\eta}^{b}\right)^{-1} R(b-\eta) G\left(\eta, \bar{x}_{\eta}^{a}\right) d \eta \\
& -a \int_{0}^{b} A\left(a I+\Gamma_{\eta}^{b}\right)^{-1} R(b-\eta) \int_{0}^{\eta} h(\eta-v) G\left(v, \bar{x}_{v}^{a}\right) d v d \eta \\
& -a \int_{0}^{b}\left(a I+\Gamma_{\eta}^{b}\right)^{-1} R(b-\eta) f(\eta) d w(\eta)-a\left(a I+\Gamma_{\eta}^{b}\right)^{-1} \sum_{k=1}^{m} R\left(b-t_{k}\right) I_{k}\left(\bar{x}_{t_{k}}^{a}\right) .
\end{aligned}
$$

By conditions (B1) and (B2), we get that the sequences $\left\{(-A)^{\beta} G\left(s, \bar{x}_{s}^{a}\right)\right\}$ and $\{f(s)\}$ are uniformly bounded on $J$. Thus there are subsequences, still denoted by $\left\{(-A)^{\beta} G\left(s, \bar{x}_{s}^{a}\right)\right\}$ and 
$\{f(s)\}$, that converge weakly to say $g(s)$ in $H$ and $f^{* *}(s)$ in $L(K, H)$, respectively. The compactness of $R(t), t>0$, implies that $R(b-s)\left[(-A)^{\beta} G\left(s, \bar{x}_{s}^{a}\right)-g(s)\right] \rightarrow 0, R(b-s)[f(s)-$ $\left.f^{* *}(s)\right] \rightarrow 0$. On the other hand, by Lemma 2.1, for all $t \in J, a\left(a I+\Gamma_{t}^{b}\right)^{-1} \rightarrow 0$ strongly as $a \rightarrow 0^{+}$and $\left\|a\left(a I+\Gamma_{t}^{b}\right)^{-1}\right\| \leq 1$. Therefore, by the Lebesgue dominated convergence theorem, it follows that

$$
\begin{aligned}
E\left\|x^{a}(b)-x_{b}\right\|_{H}^{p} & \\
\leq & 9^{p-1} E\left\|a\left(a I+\Gamma_{0}^{b}\right)^{-1}\left[E \tilde{x}_{b}-R(b)[\varphi(0)-G(0, \varphi)]-G\left(b, x_{b}^{a}\right)\right]\right\|_{H}^{p} \\
& +9^{p-1} E\left(\left\|a\left(a I+\Gamma_{\eta}^{b}\right)^{-1} \sum_{k=1}^{m} R\left(b-t_{k}\right) I_{k}\left(\bar{x}_{t_{k}}^{a}\right)\right\|_{H}\right)^{p} \\
& +9^{p-1} E\left(\int_{0}^{b}\left\|a\left(a I+\Gamma_{0}^{b}\right)^{-1} \tilde{\phi}(\eta)\right\|_{H}^{2} d \eta\right)^{p / 2} \\
& +9^{p-1}\left(\int_{0}^{b}\left\|a\left(a I+\Gamma_{\eta}^{b}\right)^{-1}(-A)^{1-\beta} R(b-\eta)\left[(-A)^{\beta} G\left(\eta, \bar{x}_{\eta}^{a}\right)-g(\eta)\right]\right\|_{H} d \eta\right)^{p} \\
& +9^{p-1} E\left(\int_{0}^{b}\left\|a\left(a I+\Gamma_{\eta}^{b}\right)^{-1}(-A)^{1-\beta} R(b-\eta) g(\eta)\right\|_{H} d \eta\right)^{p} \\
& +9^{p-1}\left(\int_{0}^{b} \int_{0}^{\eta} \| a\left(a I+\Gamma_{\eta}^{b}\right)^{-1} h(\eta-v)(-A)^{1-\beta}\right. \\
& \left.\times R(b-\eta)\left[(-A)^{\beta} G\left(v, \bar{x}_{v}^{a}\right)-g(v)\right] \|_{H} d \nu d \eta\right)^{p} \\
& +9^{p-1} E\left(\int_{0}^{b} \int_{0}^{\eta}\left\|a\left(a I+\Gamma_{\eta}^{b}\right)^{-1} h(\eta-v)(-A)^{1-\beta} R(b-\eta) g(v)\right\|_{H} d v d \eta\right)^{p} \\
& +9^{p-1} E\left(\int_{0}^{b}\left\|a\left(a I+\Gamma_{\eta}^{b}\right)^{-1} R(b-\eta)\left[f(\eta)-f^{* *}(\eta)\right]\right\|_{H}^{2} d \eta\right)^{p / 2} \\
& +9^{p-1} E\left(\int_{0}^{b}\left\|a\left(a I+\Gamma_{\eta}^{b}\right)^{-1} R(b-\eta) f^{* *}(\eta)\right\|_{H}^{2} d \eta\right)^{p / 2} \rightarrow 0 \text { as } a \rightarrow 0^{+} .
\end{aligned}
$$

So $x^{a}(b) \rightarrow x_{b}$ holds, which shows that system (1.1)-(1.3) is approximately controllable and the proof is complete.

\section{Example}

Consider the following impulsive neutral stochastic partial integro-differential inclusion with impulsive effects and infinite delays of the form:

$$
\begin{gathered}
d\left[z(t, x)-\int_{-\infty}^{t} \int_{0}^{\pi} \vartheta(s-t, \eta, x) z(s, \eta) d \eta d s\right] \\
\in \frac{\partial^{2}}{\partial^{2} t}\left[z(t, x)+\int_{0}^{t} \tilde{b}(t-s) z(s, x) d s\right] d t \\
\quad+\tilde{u}(t, x) d t+\int_{-\infty}^{t} \varpi(t, s-t, x, z(s, x)) d s d w(t), \\
0 \leq t \leq b, 0 \leq x \leq \pi, t \neq t_{k}, k=1, \ldots, m, \\
z(t, 0)=z(t, \pi)=0, \quad 0 \leq t \leq b,
\end{gathered}
$$




$$
\begin{aligned}
& \Delta z\left(t_{k}, x\right)=\int_{-\infty}^{t_{k}} \eta_{k}\left(s-t_{k}\right) z(s, x) d s, \quad k=1,2, \ldots, m, \\
& z(t, x)=\varphi(t, x), \quad t \leq 0,0 \leq x \leq \pi
\end{aligned}
$$

where $\varphi$ is continuous and $\tilde{u}:[0, b] \times(0, \pi) \rightarrow(0, \pi)$ is continuous in $t$, and there exists a constant $K_{1}$ such that $|\tilde{b}(t-s)| \leq K_{1} . w(t)$ denotes a standard cylindrical Wiener process in $H$ defined on a stochastic space $(\Omega, \mathcal{F}, P)$. Take $H=L^{2}([0, \pi])$ with the norm $\|\cdot\|$ and define the operator $A$ by $A \omega=\omega^{\prime \prime}$ with the domain

$$
D(A):=\left\{\omega \in H: \omega, \omega^{\prime} \text { are absolutely continuous, } \omega^{\prime \prime} \in H, \omega(0)=\omega(\pi)=0\right\} .
$$

Then $A$ generates a strongly continuous semigroup that is analytic, and the resolvent operator $R(t)$ can be extracted from this analytic semigroup (see [27]). Furthermore, $A$ has a discrete spectrum with eigenvalues of the form $-n^{2}, n \in \mathbb{N}$, whose corresponding (normalized) eigenfunctions are given by $z_{n}(x)=\sqrt{\frac{2}{\pi}} \sin (n x)$. In addition, the following properties hold:

(a) If $\omega \in D(A)$, then

$$
A \omega=-\sum_{n=1}^{\infty} n^{2}\left\langle\omega, z_{n}\right\rangle z_{n} .
$$

(b) For $\omega \in H$ and $\alpha \in(0,1)$,

$$
(-A)^{-\alpha} \omega=\sum_{n=1}^{\infty} \frac{1}{n^{2 \alpha}}\left\langle\omega, z_{n}\right\rangle z_{n} .
$$

(c) The operator $(-A)^{\alpha}: D\left((-A)^{\alpha}\right) \subseteq H \rightarrow H$ is given by

$$
(-A)^{\alpha} \omega=\sum_{n=1}^{\infty} n^{2 \alpha}\left\langle\omega, z_{n}\right\rangle z_{n}
$$

on the space $D\left((-A)^{\alpha}\right)=\left\{\omega(\cdot) \in H: \sum_{n=1}^{\infty} n^{2 \alpha}\left\langle\omega, z_{n}\right\rangle z_{n} \in H\right\}$.

Define an infinite dimensional control space $U$ by

$$
U=\left\{u=\sum_{n=2}^{\infty} u_{n} z_{n}(\xi) \mid \sum_{n=2}^{\infty} u_{n}^{2}<\infty\right\}
$$

with a norm defined by $\|u\|_{U}=\left(\sum_{n=2}^{\infty} u_{n}^{2}\right)^{\frac{1}{2}}$ and a linear continuous mapping $B$ from $U$ to $H$ as follows:

$$
(B u)(\xi)=2 u_{2} z_{1}(\xi)+\sum_{n=2}^{\infty} u_{n} z_{n}(\xi) \quad \text { for } u=\sum_{n=2}^{\infty} u_{n} z_{n} \in U .
$$

Obviously, $\|B\|_{L(U, H)} \leq \sqrt{5}$. On the other hand, it is easy to compute that

$$
\left(B^{*} v\right)(\xi)=\left(2 v_{1}+v_{2}\right) z_{2}(\xi)+\sum_{n=3}^{\infty} v_{n} z_{n}(\xi)
$$

with $v=\sum_{n=1}^{\infty} v_{n} z_{n} \in H$. 
Let $r \geq 0,1 \leq p<1$, and let $\tilde{h}:(-\infty,-r] \rightarrow \mathbb{R}$ be a nonnegative measurable function which satisfies the conditions (h-5), (h-6) in the terminology of Hino et al. [34]. Briefly, this means that $\tilde{h}$ is locally integrable and there is a nonnegative, locally bounded function $\gamma$ on $(-\infty, 0]$ such that $\tilde{h}(\xi+\tau) \leq \gamma(\xi) \tilde{h}(\tau)$ for all $\xi \leq 0$ and $\theta \in(-\infty,-r) \backslash N_{\xi}$, where $N_{\xi} \subseteq(-\infty,-r)$ is a set whose Lebesgue measure is zero. We denote by $\mathcal{P C} \mathcal{C}_{r} \times L^{p}(\tilde{h}, H)$ the set consisting of all classes of functions $\varphi:(-\infty, 0] \rightarrow H$ such that $\left.\varphi\right|_{[-r, 0]} \in \mathcal{P C}([-r, 0], H)$, $\varphi(\cdot)$ is Lebesgue measurable on $(-\infty,-r)$, and $\tilde{h}\|\varphi\|^{p}$ is Lebesgue integrable on $(-\infty,-r)$. The semi-norm is given by

$$
\|\varphi\|_{\mathcal{B}}=\sup _{-r \leq \tau \leq 0}\|\varphi(\tau)\|+\left(\int_{-\infty}^{-r} \tilde{h}(\tau)\|\varphi\|^{p} d \tau\right)^{1 / p} .
$$

The space $\mathcal{B}=\mathcal{P C}_{r} \times L^{p}(\tilde{h}, H)$ satisfies axioms (A)-(C). Moreover, when $r=0$ and $p=$ 2, we can take $\tilde{H}=1, M(t)=\gamma(-t)^{1 / 2}$ and $K(t)=1+\left(\int_{-t}^{0} \tilde{h}(\tau) d \tau\right)^{1 / 2}$ for $t \geq 0$ (see [34, Theorem 1.3.8] for details).

Additionally, we will assume that:

(i) The functions $\vartheta(s, \eta, x), \frac{\partial \vartheta(s, \eta, x)}{\partial x}$ are measurable, $\vartheta(s, \eta, \pi)=\vartheta(s, \eta, 0)=0$ and

$$
L_{\vartheta}=\max \left\{\left(\int_{0}^{\pi} \int_{-\infty}^{0} \int_{0}^{\pi} \frac{1}{\tilde{h}(s)}\left(\frac{\partial^{i} \vartheta(s, \eta, x)}{\partial^{i} x}\right)^{2} d \eta d s d x\right)^{\frac{1}{2}}: i=0,1\right\}<\infty .
$$

(ii) The function $\varpi: \mathbb{R}^{4} \rightarrow \mathbb{R}$ is continuous, and there exist continuous functions $a_{1}, a_{2}: \mathbb{R} \rightarrow \mathbb{R}$ such that

$$
|\varpi(t, s, x, y)| \leq a_{1}(t) a_{2}(s)|y|, \quad(t, s, x, y) \in \mathbb{R}^{4}
$$

with $L_{\varpi}=\left(\int_{-\infty}^{0} \frac{\left(a_{2}(s)\right)^{2}}{\tilde{h}(s)} d s\right)^{\frac{1}{2}}<\infty$.

(iii) The functions $\eta_{k}: \mathbb{R} \rightarrow \mathbb{R}, k=1, \ldots, m$, are continuous and $\tilde{L}_{k}=\left(\int_{-\infty}^{0} \frac{\left(\eta_{k}(s)\right)^{2}}{\tilde{h}(s)} d s\right)^{\frac{1}{2}}<\infty$ for every $k=1,2, \ldots, m$.

Take $\varphi \in \mathcal{B}=\mathcal{P C}_{0} \times L^{2}(\tilde{h}, H)$ with $\psi(\theta)(x)=\psi(\theta, x),(\theta, x) \in(-\infty, 0] \times \mathcal{B}$. Let $z(s)(x)=$ $z(s, x) . G:[0, b] \times \mathcal{B} \rightarrow H, F:[0, b] \times \mathcal{B} \rightarrow \mathcal{P}(H)$ be the operators defined by

$$
\begin{aligned}
& G(t, \psi)(x)=\int_{-\infty}^{0} \int_{0}^{\pi} \vartheta(\theta, v, \psi(\theta)(x)) d v d \theta, \\
& F(t, \psi)(x)=\int_{-\infty}^{0} \varpi(t, \theta, x, \psi(\theta)(x)) d \theta .
\end{aligned}
$$

Also define the maps $I_{k}$ and $B$ by

$$
\begin{aligned}
& I_{k}(\psi)(x)=\int_{-\infty}^{0} \eta_{k}(\theta) \psi(\theta)(x) d \theta, \\
& (B u)(t)(x)=\tilde{u}(t, x) .
\end{aligned}
$$

Under the above assumptions, problem (5.1)-(5.4) can be written as (1.1)-(1.3). Moreover, the range of $G(\cdot)$ is contained in $H_{\frac{1}{2}},(-A)^{\frac{1}{2}} G, F, I_{k}(k=1, \ldots, m)$ are bounded linear operators with $E\left\|(-A)^{\frac{1}{2}} G\right\|_{L(\mathcal{B}, H)}^{p} \leq L_{G}, E\|F\|_{L(\mathcal{B}, H)}^{p} \leq L_{F}, E\left\|I_{k}\right\|_{L(\mathcal{B}, H)}^{p} \leq \gamma_{k}, i=1, \ldots, m$, where 
$L_{G}=\left(L_{\vartheta}\right)^{p}, L_{F}=\left(\left\|a_{1}\right\|_{\infty} L_{\varpi}\right)^{p}, \gamma_{k}=\left(\tilde{L}_{k}\right)^{p}, k=1, \ldots, m$. Further, we can impose some suitable conditions on the above-defined functions to verify the assumptions on Theorem 3.1. Therefore, assumptions (H1)-(H5), (B1) and (B2) all hold, the associated linear system of (5.1)-(5.4) is not exactly controllable but it is approximately controllable. Hence, by Theorems 3.1 and 4.1, system (5.1)-(5.4) is approximately controllable on $[0, b]$.

\section{Conclusion}

In this paper, we have studied the approximate controllability of first-order impulsive partial neutral stochastic functional integro-differential inclusions with infinite delay (1.1)(1.3) in a separable real Hilbert space. Through the nonlinear alternative of Leray-Schauder type for multivalued maps due to O'Regan under the compactness assumption, we investigated the sufficient conditions for the approximate controllability of the system; more precisely, by using the fixed point theorem, analytic resolvent operators, fractional powers of closed operators combined with approximation techniques. The conditions, under which the approximate controllability of the nonlinear impulsive stochastic system is implied by the approximate controllability of its corresponding linear part and the jump operators $I_{k}$ $(k=1, \ldots, m)$ are continuous, have been formulated and proved. Finally, an application has been provided to illustrate the applicability of the new result.

In the future work we are going to study the approximate controllability of second-order and fractional impulsive partial stochastic functional differential inclusions with infinite delay for which the discussion may be more difficult and greatly different from the present situation.

\section{Competing interests}

The authors declare that they have no competing interests.

\section{Authors' contributions}

The authors completed the paper, and read and approved the final manuscript.

\section{Acknowledgements}

The authors would like to thank the editor and the reviewers for their constructive comments and suggestions. This work is supported by the National Natural Science Foundation of China (Grant No. 11461019) and is supported by the President Found of Scientific Research Innovation and Application of Hexi University (Grant No. xz2013-10).

Received: 9 October 2014 Accepted: 8 January 2015 Published online: 31 March 2015

References

1. Bashirov, AE, Mahmudov, NI: On concepts of controllability for deterministic and stochastic systems. SIAM J. Control Optim. 37, 1808-1821 (1999)

2. Klamka, J: Constrained approximate controllability. IEEE Trans. Autom. Control 45, 1745-1749 (2000)

3. Balasubramaniam, P, Ntouyas, SK: Controllability for neutral stochastic functional differential inclusions with infinite delay in abstract space. J. Math. Anal. Appl. 324, 161-176 (2006)

4. Sakthivel, R, Ganesh, R, Suganya, S: Approximate controllability of fractional neutral stochastic system with infinite delay. Rep. Math. Phys. 70, 291-311 (2012)

5. Sakthivel, R, Ganesh, R, Ren, Y, Anthoni, SM: Approximate controllability of nonlinear fractional dynamical systems. Commun. Nonlinear Sci. Numer. Simul. 18, 3498-3508 (2013)

6. Muthukumar, P, Rajivganthi, C: Approximate controllability of fractional order neutral stochastic integro-differential system with nonlocal conditions and infinite delay. Taiwan. J. Math. 17, 1693-1713 (2013)

7. Triggiani, R: A note on the lack of exact controllability for mild solutions in Banach spaces. SIAM J. Control Optim. 15 407-411 (1977)

8. Mahmudov, NI, Denker, A: On controllability of linear stochastic systems. Int. J. Control 73, 144-151 (2000)

9. Dauer, JP, Mahmudov, NI: Controllability of stochastic semilinear functional differential equations in Hilbert spaces. J. Math. Anal. Appl. 290, 373-394 (2004)

10. Benchohra, M, Henderson, J, Ntouyas, SK: Impulsive Differential Equations and Inclusions. Hindawi Publishing Corporation, New York (2006)

11. Sakthivel, R, Mahmudov, NI, Kim, JH: Approximate controllability of nonlinear impulsive differential systems. Rep. Math. Phys. 60, 85-96 (2007)

12. Ganesh, R, Sakthivel, R, Mahmudov, Nl: Approximate controllability of fractional functional equations with infinite delay. Topol. Methods Nonlinear Anal. 43, 345-364 (2014) 
13. Sakthivel, $R$, Anandhi, ER, Lee, SG: Approximate controllability of impulsive differential inclusions with nonlocal conditions. Dyn. Syst. Appl. 18, 637-654 (2009)

14. Fu, X: Approximate controllability for neutral impulsive differential inclusions with nonlocal conditions. J. Dyn. Control Syst. 17, 359-386 (2011)

15. Anguraj, A, Vinodkumar, A: Existence, uniqueness and stability results of impulsive stochastic semilinear neutral functional differential equations with infinite delays. Electron. J. Qual. Theory Differ. Equ. 2009, 67 (2009)

16. Lin, A, Ren, Y, Xia, N: On neutral impulsive stochastic integro-differential equations with infinite delays via fractional operators. Math. Comput. Model. 51, 413-424 (2010)

17. Yan, Z, Yan, X: Existence of solutions for impulsive partial stochastic neutral integrodifferential equations with state-dependent delay. Collect. Math. 64, 235-250 (2013)

18. Sakthivel, R, Nieto, JJ, Mahmudov, NI: Approximate controllability of nonlinear deterministic and stochastic systems with unbounded delay. Taiwan. J. Math. 14, 1777-1797 (2010)

19. Sakthivel, R, Ren, Y, Mahmudov, NI: Approximate controllability of second-order stochastic differential equations with impulsive effects. Mod. Phys. Lett. B 24, 1559-1572 (2010)

20. Subalakshmi, R, Balachandran, K: Approximate controllability of nonlinear stochastic impulsive integrodifferential systems in Hilbert spaces. Chaos Solitons Fractals 42, 2035-2046 (2009)

21. Shen, L, Sun, J: Approximate controllability of stochastic impulsive functional systems with infinite delay. Automatica 48, 2705-2709 (2012)

22. Zang, $Y, L i, J:$ Approximate controllability of fractional impulsive neutral stochastic differential equations with nonlocal conditions. Bound. Value Probl. 2013, 193 (2013)

23. Lin, $\mathrm{A}, \mathrm{Hu}$, L: Existence results for impulsive neutral stochastic functional integro-differential inclusions with nonlocal initial conditions. Comput. Math. Appl. 59, 64-73 (2010)

24. Yan, Z, Yan, X: Existence of solutions for a impulsive nonlocal stochastic functional integrodifferential inclusion in Hilbert spaces. Z. Angew. Math. Phys. 64, 573-590 (2013)

25. Ren, Y, Hu, L, Sakthivel, R: Controllability of impulsive neutral stochastic functional differential inclusions with infinite delay. J. Comput. Appl. Math. 235, 2603-2614 (2011)

26. Grimmer, R: Resolvent operators for integral equations in a Banach space. Trans. Am. Math. Soc. 273, 333-349 (1982)

27. Grimmer, R, Pritchard, AJ: Analytic resolvent operators for integral equations in a Banach space. J. Differ. Equ. 50, 234-259 (1983)

28. Hale, JK, Kato, J: Phase spaces for retarded equations with infinite delay. Funkc. Ekvacioj 21, 11-41 (1978)

29. Hernández, E, Pierri, M, Goncalves, G: Existence results for an impulsive abstract partial differential equation with state-dependent delay. Comput. Math. Appl. 52, 411-420 (2006)

30. Fitzpatrick, PM, Petryshyn, WV: Fixed point theorems for multivalued noncompact acyclic mappings. Pac. J. Math. 54, $17-23(1974)$

31. Da Prato, G, Zabczyk, J: Stochastic Equations in Infinite Dimensions. Cambridge University Press, Cambridge (1992)

32. O'Regan, D: Nonlinear alternatives for multivalued maps with applications to operator inclusions in abstract spaces. Proc. Am. Math. Soc. 127(12), 3557-3564 (1999)

33. Lasota, A, Opial, Z: An application of the Kakutani-Ky-Fan theorem in the theory of ordinary differential equations. Bull. Acad. Pol. Sci., Sér. Sci. Math. Astron. Phys. 13, 781-786 (1965)

34. Hino, Y, Murakami, S, Naito, T: Functional-Differential Equations with Infinite Delay. Lecture Notes in Mathematics, vol. 1473. Springer, Berlin (1991)

\section{Submit your manuscript to a SpringerOpen ${ }^{\circ}$ journal and benefit from:}

- Convenient online submission

Rigorous peer review

- Immediate publication on acceptance

- Open access: articles freely available online

- High visibility within the field

- Retaining the copyright to your article 